\title{
A discontinuous Galerkin reduced basis numerical homogenization method for fluid flow in porous media
}

\author{
Assyr Abdulle, Ondrej Budáč
}

\begin{abstract}
We present a new conservative multiscale method for Stokes flow in heterogeneous porous media. The method couples a discontinuous Galerkin finite element method (DG-FEM) at the macroscopic scale for the solution of an effective Darcy equation with a Stokes solver at the pore scale to recover effective permeabilities at macroscopic quadrature points. To avoid costly computation of numerous Stokes problems throughout the macroscopic computational domain, the pore geometry is parametrized and a model order reduction algorithm is used to select representative microscopic geometries. Accurate Stokes solutions and related permeabilities are obtained for these representative geometries in an offline stage. In an online stage, the DG-FEM is computed with permeabilities recovered at the desired macroscopic quadrature points from the precomputed Stokes solutions. The multiscale method is shown to be mass conservative at the macro scale and the computational cost for the online stage is similar to the cost of solving a single scale Darcy problem. Numerical experiments for two and three dimensional problems illustrate the efficiency and the performance of the proposed method.
\end{abstract}

Keywords. Stokes flow, Darcy equation, numerical homogenization, reduced basis, discontinuous Galerkin, mass conservation

AMS subject classifications. 65N30, 76D07, 74Qxx, 35Q86

\section{Introduction}

Fluid flow in porous media plays an important role in many scientific and engineering applications. The effective flow is commonly described by the Darcy equation, where the porous structure is accounted for by an effective permeability tensor. While this effective permeability can be modeled from macroscopic experiments for some applications, it is desirable in many situations to have a more fundamental description of permeability that relies on a (microscopic) pore scale. At this scale, the velocity of the fluid flows around solid obstacles is modeled by the Stokes equation. However, solving the Stokes equation at pore scale for a macroscopic computational domain has a prohibitive computational cost. We focus here on models and numerical methods that bridge the Darcy and the Stokes model and combine them into a multiscale method. Such methods are typically derived from the homogenization theory $[9,22,24]$ that studies the limit of the fine scale Stokes model when the pore size tends to zero. The homogenization theory provides a methodology to obtain an effective permeability from the local pore geometry by solving microscopic Stokes problems.

Multiscale method coupling Darcy and Stokes solvers have been considered recently. In [3], the Darcy-Stokes finite element heterogeneous multiscale method (DS-FE-HMM) has been introduced. The coupling strategy is based on the heterogeneous multiscale method (HMM), reviewed in [6]. The Darcy problem is solved using the finite element method on a macroscopic mesh, where the size of the elements is not restricted by the pore scale size $\varepsilon$. The effective permeability is recovered around each quadrature point of the macroscopic mesh by sampling the local porous structure and solving the Stokes micro problems numerically. The average of the microscopic velocity fields permits then to infer a macroscopic permeability at the macro quadrature points. By solving the Stokes problems only on small domains around macroscopic quadrature points, this coupling strategy is much more efficient than solving a Stokes problem at pore scale on the whole macroscopic domain. However, the large number of Stokes problems to be solved (whose number increases with a macroscopic mesh refinement) makes this strategy still computationally expensive, specially for three-dimensional problems. 
This issue has been addressed in [5] by employing a reduced basis method at the micro scale that avoids repeated computation of microscopic Stokes problems through the selection of representative microscopic geometries in an offline stage.

Other numerical methods that rely on the Stokes equation at pore scale have been proposed in $[10,13,23]$. The multiscale approach in [10] uses the control volume method to discretize the Darcy equation and a Navier-Stokes model is used on the micro scale. While this approach allows a conservative macro solver and more precise micro model, there are some limiting assumptions: piecewise periodic micro structure, alignment of the micro structure to a coarse grid, and no volumetric forces. Another method, the multiscale FEM [13] uses a hierarchy of macroscopic grids where micro problems are solved with various accuracy. This method is efficient for simple macroscopic domains (such as squares or a union of squares) but more difficult to use for complicated macroscopic domains. It also not mass conservative at the macro scale.

None of the reviewed multiscale methods for Stokes flow in porous can simultaneously accommodate:

- higher-order macroscopic methods on arbitrary macro domains,

- fast and accurate resolution of the micro scale,

- conservation of mass.

In this paper we present a new multiscale method that addresses theses three goals. At the macro scale we use a symmetric interior penalty discontinuous Galerkin finite element method (SIP-DG-FEM) with numerical quadrature. At each quadrature point of the macro mesh we approximate the local permeability by a microscopic solver. The microscopic computation of the permeability relies on the reduced basis technique developed in [5]. We next briefly describe the approach on the macro and the micro scale.

The macroscopic method, the SIP-DG-FEM (see [11] for a reference), uses a discontinuous finite element space, where continuity of the solution and Dirichlet boundary conditions are imposed weakly via penalty terms on the edge jumps. This macroscopic method has been successfully applied in the multiscale context for elliptic problems [2]. The SIP-DG-FEM is further consistent and conservative when applied to single scale problems and these properties are inherited by our multiscale method.

The effective permeability that needs to be approximated at every macroscopic quadrature point is computed as follows. We assume that the local porous geometry is known for any macroscopic coordinate and further, it can be mapped to a reference micro geometry. This allows us to map the Stokes micro problems to the reference domain, with parameter dependent coefficients. A fine micro mesh is introduced in the reference micro geometry and a stable finite element pair is used to discretize the micro problems. We next discuss a reduced basis $(\mathrm{RB})$ algorithm that is divided into offline and online two stages. The offline stage can be costly but it is performed only once. In the offline phase we construct the RB solution space that is spanned by a small number of micro solutions that are solved in the reference micro mesh for a small set of macroscopic parameters chosen via a greedy algorithm. We use a Petrov-Galerkin projection of the micro problem (see [4]), where the reduced test space is parameter-dependent. This choice guarantees approximation and algebraic stability of the $\mathrm{RB}$ method. The online phase can be used repeatedly for any macroscopic coordinate. We use the precomputed values to quickly assemble a small, dense stiffness matrix and right hand side for the reduced problem. Then, the effective permeability can be approximated. The cost of the online phase is independent of the reference microscopic meshes.

We discuss the well-posedness and a priori error analysis of the multiscale method. In the error analysis we use the classical decomposition of error depending on its source: the macro, micro, and RB error. To achieve an optimal method, all three errors should be balanced. This is also studied via numerical experiments.

This paper is structured as follows. In Section 2 we present the multiscale flow problem and recall some homogenization results. We describe the numerical multiscale method in Section 3 and provide analysis in Section 4. Two and three-dimensional experiments that corroborate the theoretical results and illustrate the performance of our method are provided in Section 5.

Notation. Let $C$ denote a generic constant whose value can change at any occurrence but it depends only on explicitly indicated quantities. We consider a domain $\Omega \subset \mathbb{R}^{d}, d \in \mathbb{N}$ and the usual Lebesgue space $L^{p}(\Omega)$ and Sobolev space $W^{k, p}(\Omega)$ equipped with the usual norms $\|\cdot\|_{L^{p}(\Omega)}$ and $\|\cdot\|_{W^{k, p}(\Omega)}$. On the factor space $L^{2}(\Omega) / \mathbb{R}$, we define $\|q\|_{L^{2}(\Omega) / \mathbb{R}}=$ 
$\inf _{s \in \mathbb{R}}\|q+s\|_{L^{2}(\Omega)}$. For $p=2$ we apply the Hilbert space notation $H^{k}(\Omega)$ and $H_{0}^{1}(\Omega)$ and define the seminorm $|q|_{H^{1}(\Omega)}=\left(\sum_{i=1}^{d}\left\|\partial_{i} q\right\|_{L^{2}(\Omega)}^{2}\right)^{1 / 2}$. The standard scalar product on $L^{2}(\Omega)$ is denoted by $(\cdot, \cdot)_{L^{2}(\Omega)}$. Given a matrix $A \in \mathbb{R}^{d \times d}$ with entries $A_{i j}$, we denote its Frobenius norm by $\|A\|_{\mathrm{F}}=\left(\sum_{i, j=1}^{d} A_{i j}^{2}\right)^{1 / 2}$. Given a vector $\xi \in \mathbb{R}^{d}$ with entries $\xi_{i}$, we define $|\xi|=\left(\sum_{i=1}^{d} \xi_{i}^{2}\right)^{1 / 2}$.

\section{Problem setting and homogenization}

Let $d \in\{2,3\}$ and $\Omega \subset \mathbb{R}^{d}$ be a connected, bounded domain. We consider a porous geometry $\Omega_{\varepsilon} \subset \Omega$, where $\varepsilon>0$ is the size of the pore scale. The aim of the proposed numerical method is to approximate the velocity $\mathbf{u}^{\varepsilon}$ and the pressure $p^{\varepsilon}$ of the fine scale Stokes problem

$$
\begin{aligned}
-\Delta \mathbf{u}^{\varepsilon}+\nabla p^{\varepsilon}=\mathbf{f} & \text { in } \Omega_{\varepsilon}, \\
\operatorname{div} \mathbf{u}^{\varepsilon}=0 & \text { in } \Omega_{\varepsilon} .
\end{aligned}
$$

We do not attempt to solve (1) directly as the cost of a standard discretization of $\Omega_{\varepsilon}$ can be prohibitive for realistic $\varepsilon$, even on modern supercomputers. Instead, we rely on homogenization theory that studies the behavior of $p^{\varepsilon}$ and $\mathbf{u}^{\varepsilon}$ for $\varepsilon \rightarrow 0^{+}$. An effective limit solution can be derived in various situations, for example, for periodic porous media $[9,22,24]$ or locally periodic porous media [14], as follows. First, the solution $\left(\mathbf{u}^{\varepsilon}, p^{\varepsilon}\right)$ is extended to the whole domain $\Omega$ and denoted $\left(\mathbf{U}^{\varepsilon}, P^{\varepsilon}\right)$. Second, one can show that there exist a homogenized pressure $p^{0}$ and a homogenized velocity $\mathbf{u}^{0}$ such that $P^{\varepsilon} \rightarrow p^{0}$ strongly in $L_{\text {loc }}^{2}(\Omega) / \mathbb{R}$ and $\mathbf{U}^{\varepsilon} / \varepsilon^{2} \rightarrow \mathbf{u}^{0}$ weakly in $L^{2}(\Omega)$. Finally, $p^{0}$ can be shown to be a solution to the following Darcy problem

$$
\nabla \cdot a^{0}\left(\mathbf{f}-\nabla p^{0}\right)=0 \quad \text { in } \Omega
$$

and $\mathbf{u}^{0}=a^{0}\left(\mathbf{f}-\nabla p^{0}\right)$, where the effective permeability $a^{0}$ that is related to the porous structure of $\Omega_{\varepsilon}$ as is presented below.

Non-periodic porous media. Denote by $Y$ the $d$-dimensional unit cube $(-1 / 2,1 / 2)^{d}$ and let $Y_{\mathrm{S}} \subset \bar{Y}$ be a closed set with positive measure. Define $Y_{\mathrm{F}}=Y \backslash Y_{\mathrm{S}}$ and assume that $Y_{\mathrm{F}}$ also has a positive measure. The subscripts $\mathrm{F}$ and $\mathrm{S}$ stand for the fluid and solid part, respectively. Homogenization theory assumes that both sets $Y_{\mathrm{F}}$ and $\mathbb{R}^{d} \backslash \cup_{z \in \mathbb{Z}^{d}}\left(z+Y_{\mathrm{S}}\right)$ are connected and have locally Lipschitz boundaries. If $\Omega \subset \mathbb{R}^{d}$ and $\varepsilon>0$ are given, one can define a periodic porous medium by $\Omega_{\varepsilon}=\Omega \backslash \cup_{k \in \mathbb{Z}^{d}} \varepsilon\left(k+Y_{\mathrm{S}}\right)$.

A natural generalization of the periodic porous medium is to introduce variation in the porosity by considering a family of deformations of the reference porous geometry $\left(Y_{\mathrm{F}}, Y_{\mathrm{F}}\right)$. Consider a continuous map $\varphi: \mathbb{R}^{d} \times \bar{Y} \rightarrow \bar{Y}$ such that for every $x \in \mathbb{R}^{d}$ the map $\varphi(x, \cdot)$ : $\bar{Y} \rightarrow \bar{Y}$ is a homeomorphism such that $\varphi(x, \cdot), \varphi(x, \cdot)^{-1} \in W^{1, \infty}(Y)$. For any $x \in \Omega$ we define the local pore geometry as $Y_{\mathrm{S}}^{x}=\varphi\left(x, Y_{\mathrm{S}}\right)$ and $Y_{\mathrm{F}}^{x}=Y \backslash Y_{\mathrm{S}}^{x}$. We define the periodic porous medium $\Omega_{\varepsilon}$ for any given $\varepsilon>0$ as

$$
\Omega_{\varepsilon}=\Omega \backslash \cup_{k \in \mathbb{Z}^{d}} \varepsilon\left(k+Y_{\mathrm{S}}^{\epsilon k}\right) .
$$

For an illustration see Figure 1.

Homogenized tensor $a^{0}$. For any point $x \in \Omega$ we can compute $a^{0}(x)$ by solving Stokes micro problems and taking an integral of the computed velocity fields. For every $i \in\{1, \ldots, d\}$ find the velocity $\mathbf{u}^{i, x}$ and pressure $p^{i, x}$ such that

$$
\begin{aligned}
& -\Delta \mathbf{u}^{i, x}+\nabla p^{i, x}=\mathbf{e}^{i} \quad \text { in } Y_{\mathrm{F}}^{x}, \quad \mathbf{u}^{i, x}=0 \quad \text { on } \partial Y_{\mathrm{S}}^{x}, \\
& \operatorname{div} \mathbf{u}^{i, x}=0 \quad \text { in } Y_{\mathrm{F}}^{x}, \quad \mathbf{u}^{i, x} \text { and } p^{i, x} \quad \text { are } Y \text {-periodic, }
\end{aligned}
$$

where $\mathbf{e}^{i}$ is the $i$-th canonical basis vector in $\mathbb{R}^{d}$. The pressure is unique only up to a constant (cf. section 3.2). We then define

$$
a_{i j}^{0}(x)=\int_{Y_{\mathrm{F}}^{x}} \mathbf{u}_{i}^{j, x} \mathrm{~d} y \quad \forall i, j \in\{1, \ldots, d\} .
$$

\section{The multiscale numerical method}

We present a new numerical method for solving the effective macro problem (2) coupled with the micro problems (4) that are used to approximate the effective permeability (5). The 


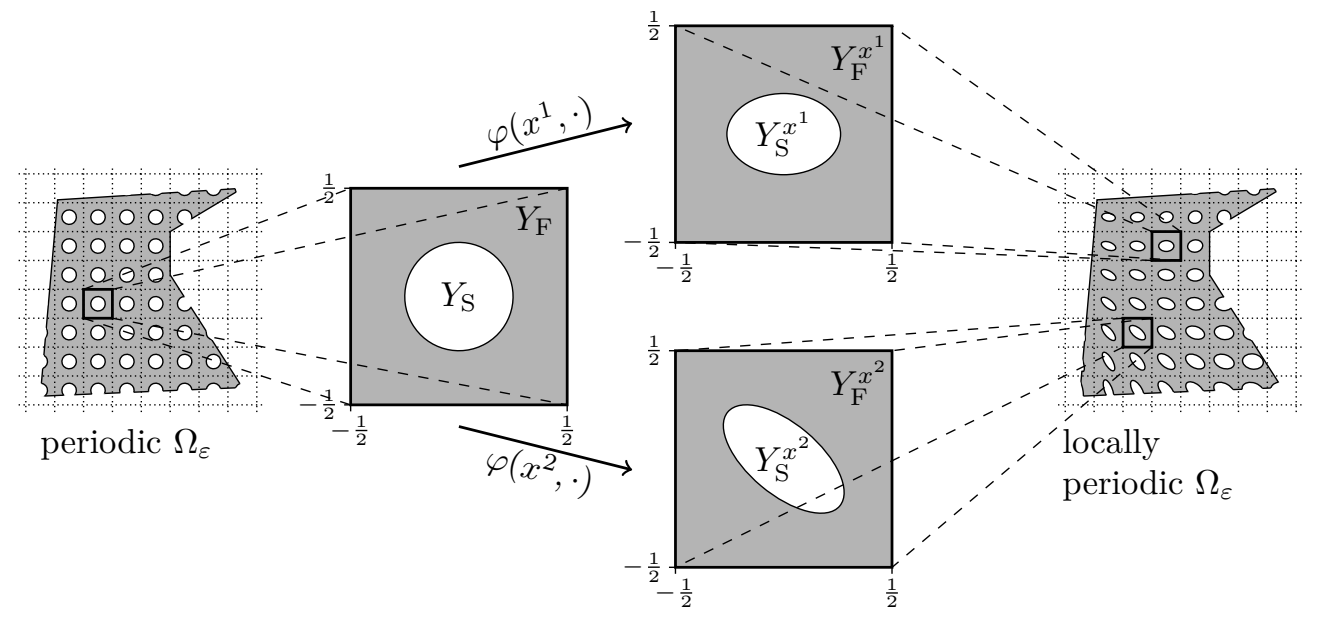

Figure 1: A reference porous geometry $\left(Y_{\mathrm{F}}, Y_{\mathrm{S}}\right)$, where $Y_{\mathrm{S}}$ represents a circular solid inclusion. A periodic porous medium (left) and a non-periodic porous medium (right) with a zoom on the local porous geometry $\left(Y_{\mathrm{F}}^{x}, Y_{\mathrm{S}}^{x}\right)$ at two points $x^{1}, x^{2} \in \Omega$.

macro problem (2) is discretized using the symmetric interior penalty (SIP) discontinuous Galerkin finite element method (DG-FEM) with numerical quadrature (see section 3.1). In section 3.2 we describe a finite element (FE) discretization of the micro problems (4) and we define a reduced basis $(\mathrm{RB})$ method that gives an approximation $a^{\mathrm{RB}}(x) \approx a^{0}(x)$ for any $x \in \Omega$. An efficient implementation of the RB method is presented in section 3.3, where an offline/online splitting is described. Finally, we apply the heterogeneous multiscale method (HMM) framework (see [6]) by using the computable tensor $a^{\mathrm{RB}}$ (instead of $a^{0}$ ) in the DGFEM and thus introduce a fully discrete multiscale method in section 3.4.

3.1. Macro scale: The symmetric interior penalty DG-FEM. We begin by discretizing the macro scale and for the moment we assume that the effective permeability $a^{0}(x)$ is known for every $x \in \Omega$. Such macro problem discretization was already studied in the case of homogenization-based multiscale methods for elliptic problems [2,7].

While the macroscopic Darcy equation (2) follows directly from the homogenization theory, one usually considers the form

$$
\begin{aligned}
-\nabla \cdot\left(a^{0} \nabla p^{0}\right) & =f & & \text { in } \Omega, \\
p^{0} & =g_{\mathrm{D}} & & \text { on } \Gamma_{\mathrm{D}}, \\
a^{0} \nabla p^{0} \cdot \mathbf{n} & =g_{\mathrm{N}} & & \text { on } \Gamma_{\mathrm{N}},
\end{aligned}
$$

where the boundary is divided into a Dirichlet and Neumann part by $\partial \Omega=\Gamma_{\mathrm{D}} \cup \Gamma_{\mathrm{N}}$ and $\mathbf{n}$ denotes the outward normal vector.

Let $\mathcal{T}_{H}$ be a conformal, shape-regular partition of $\Omega$ into simplicial elements $K$ of diameter $H_{K}$ with $H=\max _{K \in \mathcal{T}_{H}} H_{K}$. Let $\mathcal{E}_{\text {int }}$ be the set of all interior element interfaces (edges or faces), $\mathcal{E}_{\mathrm{D}}$ the set of all boundary Dirichlet interfaces, and $\mathcal{E}_{\mathrm{N}}$ the set of all boundary Neumann interfaces. Finally, define $\mathcal{E}=\mathcal{E}_{\text {int }} \cup \mathcal{E}_{\mathrm{D}} \cup \mathcal{E}_{\mathrm{N}}$ and $\mathcal{E}_{\text {pen }}=\mathcal{E}_{\text {int }} \cup \mathcal{E}_{\mathrm{D}}$. Let $e \in \mathcal{E}_{\text {int }}$ be an interface between two elements $K^{1}$ and $K^{2}$ and let $\mathbf{n}^{i}$ denote the outward normal vector of $K^{i}$ on $e$ for $i \in\{1,2\}$. Let $q$ be any element-wise smooth function and $\mathbf{v}$ be any element-wise smooth vector function. For $i \in\{1,2\}$, let $q^{i}$ and $\mathbf{v}^{i}$ denote the trace of $q$ and $\mathbf{v}$ on $e$ from within $K^{i}$, respectively. We define the average $\{\cdot\}$ and the jump $\llbracket \cdot \rrbracket$ of $q$ and $\mathbf{v}$ over $e$ by

$$
\begin{aligned}
& \{q\}=\left(q^{1}+q^{2}\right) / 2, \quad \llbracket q \rrbracket=q^{1} \mathbf{n}^{1}+q^{2} \mathbf{n}^{2}, \\
& \{\mathbf{v}\}=\left(\mathbf{v}^{1}+\mathbf{v}^{2}\right) / 2, \quad \llbracket \mathbf{v} \rrbracket=\mathbf{v}^{1} \cdot \mathbf{n}^{1}+\mathbf{v}^{2} \cdot \mathbf{n}^{2} .
\end{aligned}
$$

For any boundary edge $e \in \mathcal{E}_{\mathrm{D}} \cup \mathcal{E}_{\mathrm{N}}$, belonging only to one element $K^{1}$, we define $\{q\}=q^{1}$, $\llbracket q \rrbracket=q^{1} \mathbf{n}^{1},\{\mathbf{v}\}=\mathbf{v}^{1}$, and $\llbracket \mathbf{v} \rrbracket=\mathbf{v}^{1} \cdot \mathbf{n}^{1}$. Let us define a discontinuous finite element space of degree $l \in \mathbb{N}$ by

$$
S_{\mathrm{dis}}^{l}\left(\mathcal{T}_{H}\right)=\left\{q^{H} \in L^{2}(\Omega) ;\left.q^{H}\right|_{K} \in \mathcal{P}^{l}(K), \forall K \in \mathcal{T}_{H}\right\},
$$

where $\mathcal{P}^{l}(K)$ is the space of polynomials on the simplicial element $K$ of total degree $l$. 
SIP-DG-FEM derivation. We briefly recall how a symmetric interior penalty discontinuous Galerkin finite element method can be derived for the problem (6). For further details and analysis see $[2,11,16]$. We multiply the equation from (6) by a smooth test function $q$ and integrate by parts over and element $K \in \mathcal{T}_{H}$ to get

$$
\int_{K} a^{0} \nabla p^{0} \cdot \nabla q \mathrm{~d} x-\int_{\partial K} a^{0} \nabla p^{0} \cdot \mathbf{n} q \mathrm{~d} s=\int_{K} f q \mathrm{~d} x .
$$

Summing over all elements $K \in \mathcal{T}_{H}$ and using the notation (7) and the Neumann boundary condition we obtain

$$
\int_{\Omega} a^{0} \nabla p^{0} \cdot \nabla q \mathrm{~d} x-\sum_{e \in \mathcal{E}_{\mathrm{pen}}} \int_{e}\left\{a^{0} \nabla p^{0}\right\} \cdot \llbracket q \rrbracket \mathrm{d} s=\int_{\Omega} f q \mathrm{~d} x+\int_{\Gamma_{\mathrm{N}}} g_{\mathrm{N}} q \mathrm{~d} s .
$$

Notice that since $p^{0} \in H^{1}(\Omega)$, we have $\llbracket p^{0} \rrbracket=0$ on $\mathcal{E}_{\text {int }}$. Moreover the Dirichlet boundary conditions imply $\llbracket p^{0} \rrbracket=g_{\mathrm{D}} \mathbf{n}$ on $\Gamma_{\mathrm{D}}$. Taking this fact into account we can symmetrize the left-hand side of (8) and obtain

$$
\begin{aligned}
& \int_{\Omega} a^{0} \nabla p^{0} \cdot \nabla q \mathrm{~d} x-\sum_{e \in \mathcal{E}_{\mathrm{pen}}} \int_{e}\left(\left\{a^{0} \nabla p^{0}\right\} \cdot \llbracket q \rrbracket+\left\{a^{0} \nabla q\right\} \cdot \llbracket p^{0} \rrbracket\right) \mathrm{d} s \\
= & \int_{\Omega} f q \mathrm{~d} x+\int_{\Gamma_{\mathrm{D}}} a^{0} \nabla q \cdot \mathbf{n} g_{\mathrm{D}} \mathrm{d} s+\int_{\Gamma_{\mathrm{N}}} g_{\mathrm{N}} q \mathrm{~d} s .
\end{aligned}
$$

Finally, to stabilize the method we add penalty terms that will weakly impose continuity of the solution over $\mathcal{E}_{\text {int }}$ and also the Dirichlet boundary conditions. In section 4 we will define a non-negative weight function $\sigma: \cup_{e \in \mathcal{E}_{\mathrm{pen}}} e \rightarrow \mathbb{R}$ such that $\left.\sigma\right|_{e}$ is constant over each edge $e \in \mathcal{E}_{\text {pen }}$. Adding the penalty term then yields

$$
\begin{aligned}
& \int_{\Omega} a^{0} \nabla p^{0} \cdot \nabla q \mathrm{~d} x-\sum_{e \in \mathcal{E}_{\mathrm{pen}}} \int_{e}\left(\left\{a^{0} \nabla p^{0}\right\} \cdot \llbracket q \rrbracket+\left\{a^{0} \nabla q\right\} \cdot \llbracket p^{0} \rrbracket-\sigma \llbracket p^{0} \rrbracket \cdot \llbracket q \rrbracket\right) \mathrm{d} s \\
= & \int_{\Omega} f q \mathrm{~d} x+\int_{\Gamma_{\mathrm{D}}}\left(a^{0} \nabla q \cdot \mathbf{n}+\sigma q\right) g_{\mathrm{D}} \mathrm{d} s+\int_{\Gamma_{\mathrm{N}}} g_{\mathrm{N}} q \mathrm{~d} s .
\end{aligned}
$$

Numerical quadrature. To obtain a FEM with numerical quadrature based on (9) we need to take care of the integrals that contain $a^{0}$. For every element $K \in \mathcal{T}_{H}$ consider a quadrature formula $\left(x_{K_{j}}, \omega_{K_{j}}\right)_{j=1, \ldots, J}$, where $x_{K_{j}} \in K$ are integration points and $\omega_{K_{j}}>0$ are weights. The volume integral can be approximated directly with the quadrature formula and the following assumption is needed for optimal accuracy.

Assumption (Q). The quadrature formula is exact for polynomials of order up to $m=$ $\max (2 l-2, l)$. That is, for any $K \in \mathcal{T}_{H}$ and $q \in \mathcal{P}^{m}(K)$ we have $\int_{K} q \mathrm{~d} x=\sum_{j=1}^{J} \omega_{K_{j}} q\left(x_{K_{j}}\right)$.

To take care of the boundary and interface integrals containing $a^{0}$, we define the multiscale numerical flux [1,2]. The minimal number of quadrature points for which assumption (Q) can hold is $\left(\begin{array}{c}l+d-1 \\ d\end{array}\right)$. If we assume that $J=\left(\begin{array}{c}l+d-1 \\ d\end{array}\right)$ we can define for each element $K \in \mathcal{T}_{H}$ a unique interpolant $\Pi: C(K) \rightarrow \mathcal{P}^{l-1}(K)$ that is exact on all quadrature points: $\Pi(q)\left(x_{K_{j}}\right)=$ $q\left(x_{K_{j}}\right)$ for every $j \in\{1, \ldots, J\}$. For any tensor $a(x)$ that is defined at each quadrature point $x_{K_{j}}$ of $\mathcal{T}_{H}$, we define the interpolation operator $\Pi_{a}: S_{\mathrm{dis}}^{l-1}\left(\mathcal{T}_{H}\right)^{d} \rightarrow S_{\mathrm{dis}}^{l-1}\left(\mathcal{T}_{H}\right)^{d}$ that is given by

$$
\Pi_{a}(\mathbf{v})\left(x_{K_{j}}\right)=a\left(x_{K_{j}}\right) \mathbf{v}\left(x_{K_{j}}\right), \quad \forall K \in \mathcal{T}_{H}, \quad \forall j \in\{1, \ldots, J\} .
$$

We then replace the terms of type $a^{0} \nabla q$ in (9) by their polynomial approximations $\Pi_{a^{0}}(\nabla q)$. Hence, we define the discrete macroscopic approximation of (6) as follows.

Semi-discrete problem. Find $p^{H, 0} \in S_{\text {dis }}^{l}\left(\mathcal{T}_{H}\right)$ such that

$$
B_{H}^{0}\left(p^{H, 0}, q^{H}\right)=L_{H}^{0}\left(q^{H}\right) \quad \forall q^{H} \in S_{\text {dis }}^{l}\left(\mathcal{T}_{H}\right),
$$

where

$$
\begin{aligned}
B_{H}^{0}\left(p^{H}, q^{H}\right)= & \int_{\Omega} \Pi_{a^{0}}\left(\nabla p^{H}\right) \cdot \nabla q^{H}-\sum_{e \in \mathcal{E}_{\mathrm{pen}}} \int_{e}\left(\left\{\Pi_{a^{0}}\left(\nabla p^{H}\right)\right\} \cdot \llbracket q^{H} \rrbracket\right. \\
& \left.\quad+\left\{\Pi_{a^{0}}\left(\nabla q^{H}\right)\right\} \cdot \llbracket p^{H} \rrbracket-\sigma \llbracket p^{H} \rrbracket \cdot \llbracket q^{H} \rrbracket\right) \mathrm{d} s \\
L_{H}^{0}\left(q^{H}\right)= & \int_{\Omega} f q^{H} \mathrm{~d} x+\int_{\Gamma_{\mathrm{D}}}\left(\Pi_{a^{0}}\left(\nabla q^{H}\right) \cdot \mathbf{n}+\sigma q^{H}\right) g_{\mathrm{D}} \mathrm{d} s+\int_{\Gamma_{\mathrm{N}}} g_{\mathrm{N}} q^{H} \mathrm{~d} s .
\end{aligned}
$$


3.2. Micro scale: A reduced basis method. In the following two sections we recall the Petrov-Galerkin reduced basis (RB) method for the micro problems (4) and the output of interest (5). We first map the micro problems (4) from $Y_{\mathrm{F}}^{x}$ to the reference micro domain $Y_{\mathrm{F}}$, where we consider a stable FE approximation. Second, we apply a PetrovGalerkin projection to a low-dimensional solution space and a parameter-dependent test space, obtaining a reduced model (20), (22). An efficient implementation of this method and its properties are then discussed in section 3.3. We discuss here only the essential parts of the method. Further details are explained in $[4,5]$.

Weak formulation. Consider a weak formulation of (4) that uses a Lagrange multiplier to enforce the normalization of the pressure (cf. section 2). Given $x \in \Omega$ and any $i \in\{1, \ldots, d\}$, find $\mathbf{u}^{i, x} \in H_{0, p e r}^{1}\left(Y_{\mathrm{F}}^{x}\right)^{d}, p^{i, x} \in L_{2}\left(Y_{\mathrm{F}}^{x}\right)$, and $\lambda^{i, x} \in \mathbb{R}$ such that

$$
\begin{array}{rlrl}
\int_{Y_{\mathrm{F}}^{x}} \sum_{j=1}^{d} \nabla \mathbf{u}_{j}^{i, x} \cdot \nabla \mathbf{v}_{j} \mathrm{~d} y-\int_{Y_{\mathrm{F}}^{x}} p^{i, x} \operatorname{div} \mathbf{v} \mathrm{d} y=\int_{Y_{\mathrm{F}}^{x}} \mathbf{v}_{i} \mathrm{~d} y & & \forall \mathbf{v} \in H_{0, p e r}^{1}\left(Y_{\mathrm{F}}^{x}\right), \\
-\int_{Y_{\mathrm{F}}^{x}} q \operatorname{div} \mathbf{u}^{i, x} \mathrm{~d} y+\lambda^{i, x} \int_{Y_{\mathrm{F}}^{x}} q \mathrm{~d} y=0 & & \forall q \in L_{2}\left(Y_{\mathrm{F}}^{x}\right), \\
\kappa \int_{Y_{\mathrm{F}}^{x}} p^{i, x} \mathrm{~d} y=0 & \forall \kappa \in \mathbb{R} .
\end{array}
$$

The space $H_{0, p e r}^{1}\left(Y_{\mathrm{F}}^{x}\right)$ consists of $Y$-periodic functions with zero trace on $\partial Y_{\mathrm{S}}^{x}$.

Reference micro domain. We map the problem (11) from the domain $Y_{\mathrm{F}}^{x}$ to the reference domain $Y_{\mathrm{F}}$ using the change of variables $y_{\text {old }}=\varphi\left(x, y_{\text {new }}\right)$, stemming from the mapping $\varphi(x, \cdot): Y_{\mathrm{F}} \rightarrow Y_{\mathrm{F}}^{x}$. Let us denote the Jacobian $J^{x}(y)=\nabla_{y} \varphi(x, y)$ and define $\nu^{x}(y)=\operatorname{det}\left(J^{x}\right)\left(\left(J^{x}\right)^{\top} J^{x}\right)^{-1}$ and $\kappa^{x}(y)=\operatorname{det}\left(J^{x}\right)\left(J^{x}\right)^{-\top}$. The mentioned change of variables transforms (11) into

$$
\begin{aligned}
a\left(\mathbf{u}^{i, x, \mathrm{e}}, \mathbf{v} ; x\right)+b\left(\mathbf{v}, p^{i, x, \mathrm{e}} ; x\right) & =g^{i}(\mathbf{v} ; x), & & \forall \mathbf{v} \in H_{0, p e r}^{1}\left(Y_{\mathrm{F}}\right), \\
b\left(\mathbf{u}^{i, x, \mathrm{e}}, q ; x\right)+c\left(q, \lambda^{i, x, \mathrm{e}} ; x\right) & =0, & & \forall q \in L_{2}\left(Y_{\mathrm{F}}\right), \\
c\left(p^{i, x, \mathrm{e}}, \kappa ; x\right) & =0 & & \forall \kappa \in \mathbb{R},
\end{aligned}
$$

where

$$
\begin{aligned}
& a(\mathbf{u}, \mathbf{v} ; x)=\sum_{i, j=1}^{d} \int_{Y_{\mathrm{F}}} \nu_{i j}^{x}(y) \frac{\partial \mathbf{u}}{\partial y_{i}} \cdot \frac{\partial \mathbf{v}}{\partial y_{j}} \mathrm{~d} y, \quad c(q, \kappa ; x)=\kappa \int_{Y_{\mathrm{F}}} \operatorname{det}\left(J^{x}\right) q \mathrm{~d} y, \\
& b(\mathbf{v}, q ; x)=-\sum_{i, j=1}^{d} \int_{Y_{\mathrm{F}}} \kappa_{i j}^{x}(y) \frac{\partial \mathbf{v}_{i}}{\partial y_{j}} q \mathrm{~d} y, \quad g^{i}(\mathbf{v} ; x)=\int_{Y_{\mathrm{F}}} \operatorname{det}\left(J^{x}\right) \mathbf{v}_{i} \mathrm{~d} y .
\end{aligned}
$$

For the ease of manipulation, we simplify the notation of the system (12) one step further by combining the three equations into one. Let us denote $X=H_{0, p e r}^{1}\left(Y_{\mathrm{F}}\right) \times L^{2}\left(Y_{\mathrm{F}}\right) \times \mathbb{R}$. Find $\mathbf{U}_{\mathrm{e}}^{i, x}=\left(\mathbf{u}^{i, x, \mathrm{e}}, p^{i, x, \mathrm{e}}, \lambda^{i, x, \mathrm{e}}\right) \in X$ such that

$$
\begin{aligned}
& a\left(\mathbf{u}^{i, x, \mathrm{e}}, \mathbf{v} ; x\right)+b\left(\mathbf{v}, p^{i, x, \mathrm{e}} ; x\right)+b\left(\mathbf{u}^{i, x, \mathrm{e}}, q ; x\right) \\
& \begin{aligned}
\underbrace{+c\left(q, \lambda^{i, x, \mathrm{e}} ; x\right)+c\left(p^{i, x, \mathrm{e}}, \kappa ; x\right)}_{A\left(\mathbf{U}_{\mathrm{e}}^{i, x}, \mathbf{V} ; x\right)}=\underbrace{g^{i}(\mathbf{v} ; x)} \quad \forall(\mathbf{v}, q, \kappa) \in X \\
=G^{i}(\mathbf{V} ; x) \quad \forall \mathbf{V} \in X
\end{aligned}
\end{aligned}
$$

for every $\mathbf{V}=(\mathbf{v}, q, \kappa) \in X$. Here, the index e stands for exact solution. In (15) we simplified the notation further by defining a parameter dependent symmetric bilinear form $A(\cdot, \cdot ; x)$ and linear forms $G^{i}(\cdot ; x)$. The problems $(15)$ and $(11)$ are equivalent, that is, $\mathbf{u}^{i, x}(\varphi(x, y)) \equiv$ $\mathbf{u}^{i, x, \mathrm{e}}(y)$. Hence, the effective permeability (5) reads

$$
a_{i j}^{0}(x)=g^{i}\left(\mathbf{u}^{j, x}\right)=g^{i}\left(\mathbf{u}^{j, x, \mathrm{e}} ; x\right)=G^{i}\left(\mathbf{U}_{\mathrm{e}}^{j, x} ; x\right) \quad \forall i, j \in\{1, \ldots, d\} .
$$

Discretization of (15). Let $\mathcal{T}_{h}$ be a conformal, shape-regular triangulation of $Y_{\mathrm{F}}$, where $h=\max _{K \in \mathcal{T}_{h}} h_{K}$ and $h_{K}=\operatorname{diam}(K)$. We consider a stable FE pair such as the Taylor-Hood $\mathcal{P}^{k+1} / \mathcal{P}^{k} \mathrm{FE}$ given by

$$
\begin{aligned}
& L_{h}=\left\{q \in S^{k}\left(\mathcal{T}_{h}\right) ; \quad q \text { is } Y \text {-periodic }\right\}, \\
& W_{h}=\left\{\mathbf{v} \in S^{k+1}\left(\mathcal{T}_{h}\right)^{d} ; \quad \mathbf{v} \text { is } Y \text {-periodic }\right\} \text {, }
\end{aligned}
$$


where

$$
S^{k}\left(\mathcal{T}_{h}\right)=\left\{q^{h} \in L^{2}\left(Y_{\mathrm{F}}\right) ;\left.q^{h}\right|_{K} \in \mathcal{P}^{k}(K), \forall K \in \mathcal{T}_{h}\right\} .
$$

Consider a finite dimensional subspace of $X$ given by $X_{h}=W_{h} \times L_{h} \times \mathbb{R}$. We define a numerical approximation of the solution of the problem (15) and of the output of interest $a^{0}(x)$ as follows. Find $\mathbf{U}_{h}^{i, x}=\left(\mathbf{u}^{i, x, h}, p^{i, x, h}, \lambda^{i, x, h}\right) \in X_{\mathcal{N}}$ such that

$$
\begin{aligned}
A\left(\mathbf{U}_{h}^{i, x}, \mathbf{V} ; x\right) & =G^{i}(\mathbf{V} ; x) & & \forall \mathbf{V} \in X_{h}, \\
a_{i j}^{h}(x) & =G^{i}\left(\mathbf{U}_{h}^{j, x} ; x\right) & & \forall i, j \in\{1, \ldots, d\} .
\end{aligned}
$$

Petrov-Galerkin projection. Let $i \in\{1, \ldots, d\}$ and assume that we have a linear subspace $X_{i} \subset X_{h}$ that is a good approximation to the solution manifold $\mathcal{M}=\left\{\mathbf{U}_{h}^{i, x} ; x \in \Omega\right\} \subset$ $X_{h}$, i.e., a linear subspace with a small projection error with respect to the solution manifold. Our goal is to project (18) to $X_{i}$ while keeping good approximation and stability properties. It was shown in [4] that this can be achieved by considering a Petrov-Galerkin method with $X_{i}$ as a solution space and a parameter-dependent test space $Y_{i}^{x}=T\left(X_{i} ; x\right)$, where $T: X_{h} \times \Omega \rightarrow X_{h}$, called the supremizer operator, is defined below. The RB approximation of (18) then reads: find $\mathbf{U}_{\mathrm{RB}}^{i, x} \in X_{i}$ such that

$$
A\left(\mathbf{U}_{\mathrm{RB}}^{i, x}, \mathbf{V} ; x\right)=G^{i}(\mathbf{V} ; x) \quad \forall \mathbf{V} \in Y_{i}^{x} .
$$

For any $x \in \Omega$ and $\mathbf{U} \in X_{h}$ we choose $T(\mathbf{U} ; x) \in X_{h}$ such that

$$
(T(\mathbf{U} ; x), \mathbf{V})_{X}=A(\mathbf{U}, \mathbf{V} ; x) \quad \forall \mathbf{V} \in X_{h}
$$

The variational problem $(21)$ is well-posed and admits a unique solution $T(\mathbf{U} ; x)$. For any $x \in \Omega$ the operator $T(\cdot ; x): X_{h} \rightarrow X_{h}$ is linear.

Our main interest is an approximation of the effective permeability $a^{0}(x)$, which will be denoted as $a^{\mathrm{RB}}(x)$. While the straightforward choice (see $(16)$ ) would be $a_{i j}^{\mathrm{RB}}(x)=G^{i}\left(\mathbf{U}_{\mathrm{RB}}^{j, x} ; x\right)$, one can obtain higher accuracy with (see [20])

$$
a_{i j}^{\mathrm{RB}}(x)=G^{i}\left(\mathbf{U}_{\mathrm{RB}}^{j, x} ; x\right)+G^{j}\left(\mathbf{U}_{\mathrm{RB}}^{i, x} ; x\right)-A\left(\mathbf{U}_{\mathrm{RB}}^{j, x}, \mathbf{U}_{\mathrm{RB}}^{i, x} ; x\right) \quad \forall i, j \in\{1, \ldots, d\} .
$$

Selecting $Y_{i}^{x}$ as the solution space allows us to prove stability of the method, that is, the inf-sup constant number of $A(\cdot, \cdot ; x): X_{i} \times Y_{i}^{x} \rightarrow \mathbb{R}$ is not smaller than the inf-sup constant of $A(\cdot, \cdot ; x): X_{h} \times X_{h} \rightarrow \mathbb{R}$. In the next section we will give some additional details on the construction of the offline space $X_{i}$ and on the procedure to approximate $a^{0}(x)$ by $a^{\mathrm{RB}}(x)$. The full numerical multiscale method is then described in section 3.4.

3.3. Micro scale: The offline/online splitting. In this section we start with an essential ingredient of an effective reduced basis implementation: the affine decomposition of the bilinear form $A(\cdot, \cdot ; x)$ and the linear forms $G^{i}(\cdot ; x)$. We show how to construct such a decomposition provided that $\varphi(x, y)$ is piecewise linear in $y$ (an extension for a general map $\varphi(x, y)$ is outlined). We then show how the reduced basis method is split in two phases.

- The offline phase is run only once and it is used to construct the RB space $X_{i}$ and precompute necessary values for the online phase.

- The online phase can be run after the offline phase repeatedly and it provides a cheap and accurate approximation of the effective permeability $a^{\mathrm{RB}}(x)$ for any $x \in \Omega$.

It is essential that the time cost of the online phase is independent of $\operatorname{dim}\left(X_{h}\right)$, so that the cost of taking a very fine microscopic mesh will affect only the offline phase.

Affine decomposition. Let us have a closer look at the parameter-dependent linear forms $G^{i}(\cdot ; x)$ defined in (15) and (13). We have $G^{i}(\mathbf{V} ; x)=\int_{Y_{\mathrm{F}}} \operatorname{det}\left(J^{x}(y)\right) \mathbf{v}_{i}(y) \mathrm{d} y$ for every $\mathbf{V}=(\mathbf{v}, q, \kappa) \in X$. Our goal is to write $G^{i}(\cdot ; x)$ as a sum of products of functions depending only on $x$ or only on $V$. Let $R \in \mathbb{N}$ and assume that $\left\{Y_{\mathrm{F}}^{r}\right\}_{r=1}^{R}$ is a disjoint partition of $Y_{\mathrm{F}}$ such that the restriction $\left.\varphi(y ; x)\right|_{y \in Y_{\mathrm{F}}^{r}}$ is linear for every $x \in \Omega$ and $r \in\{1, \ldots, R\}$. Under this assumption the Jacobian $J^{x}(y)$ is constant for $y \in Y_{\mathrm{F}}^{r}$ (denote the constant matrix $J^{x, r}$ ) and thus we can write

$$
G^{i}(\mathbf{V} ; x)=\sum_{r=1}^{R} \operatorname{det}\left(J^{x, r}\right) \int_{Y_{\mathrm{F}}^{r}} \mathbf{v}_{i} \mathrm{~d} y .
$$

Similarly, the bilinear form $A(\cdot, \cdot ; x)$ can be decomposed into a sum of products of nonparametric bilinear forms and functions of $x$, because the coefficients $\nu_{i j}^{x}$ and $\kappa_{i j}^{x}$ are piecewise 
constant in $y$. If $\varphi(y ; x)$ is not affine, then one can apply the empirical interpolation method (see [12]) to $(x, y) \mapsto \operatorname{det}\left(J^{x}(y)\right)$ to obtain an expansion $\operatorname{det}\left(J^{x}(y)\right) \approx f_{1}(y) g_{1}(x)+\cdots+$ $f_{R}(y) g_{R}(x)$. We then have

$$
G^{i}(\mathbf{V} ; x) \approx \sum_{r=1}^{R} g_{r}(x) \int_{Y_{\mathrm{F}}} f_{r}(y) \mathbf{v}_{i} \mathrm{~d} y,
$$

where the parameter $R \in \mathbb{N}$ controls the accuracy of the approximation. Regardless of how we achieve it, we will assume for the rest of the paper that the following assumption is true.

Assumption (A). Assume that there are

- symmetric bilinear forms $A^{q}(\cdot, \cdot): X \times X \rightarrow \mathbb{R}$ for $q \in\left\{1, \ldots, Q_{A}\right\}$, where $Q_{A} \in \mathbb{N}$,

- linear forms $G^{i q}(\cdot): X \rightarrow \mathbb{R}$ for $q \in\left\{1, \ldots, Q_{G}\right\}$ and $i \in\{1, \ldots, d\}$, where $Q_{G} \in \mathbb{N}$,

- vector fields $\Theta^{A}: \Omega \rightarrow \mathbb{R}^{Q_{A}}$ and $\Theta^{G}: \Omega \rightarrow \mathbb{R}^{Q_{G}}$,

such that for any $\mathbf{U}, \mathbf{V} \in X$, parameter $x \in \Omega$, and $i \in\{1, \ldots, d\}$ we have

$$
A(\mathbf{U}, \mathbf{V} ; x)=\sum_{q=1}^{Q_{A}} \Theta_{q}^{A}(x) A^{q}(\mathbf{U}, \mathbf{V}), \quad G^{i}(\mathbf{V} ; x)=\sum_{q=1}^{Q_{G}} \Theta_{q}^{G}(x) G^{i q}(\mathbf{V}) .
$$

Based on (23) we can introduce an affine decomposition of $T(\cdot ; x)$ as

$$
T(\mathbf{U} ; x)=\sum_{q=1}^{Q_{A}} \Theta_{q}^{A}(x) T^{q}(\mathbf{U}),
$$

where the linear functionals $T^{q}: X_{h} \rightarrow X_{h}$ are defined by variational problems: Find $T^{q}(\mathbf{U}) \in X_{h}$ such that $\left(T^{q}(\mathbf{U}), \mathbf{V}\right)_{X}=A^{q}(\mathbf{U}, \mathbf{V})$ for every $\mathbf{V} \in X_{h}$.

An example of affine decomposition. To illustrate the above procedure, we give a simple example of a reference micro geometry $\left(Y_{\mathrm{F}}, Y_{\mathrm{S}}\right)$ and a piecewise-linear mapping $\varphi$ that leads to (A). Let $Y_{\mathrm{S}}$ be a circle with radius 0.25 and $Y_{\mathrm{F}}=Y \backslash Y_{\mathrm{S}}$ as is shown in Figure 2. We divide $Y_{\mathrm{F}}$ into two domain: $Y_{\mathrm{F}}^{1}=\left\{y \in Y_{\mathrm{F}} ; y_{1}<0\right\}$ and $Y_{\mathrm{F}}^{2}=\left\{y \in Y_{\mathrm{F}} ; y_{1}>0\right\}$. Given a function $r: \Omega \rightarrow(-0.5,0.5)$, the mapping $\varphi(x, \cdot): Y \rightarrow Y$ stretches the subdomains $Y_{\mathrm{F}}^{1}$ and $Y_{\mathrm{F}}^{2}$ such that their common boundary (line given by $y_{1}=0$ ) is moved to the line given by $y_{1}=r(x)$ (see Figure 2). Formally, we have

$$
\varphi(x, y)= \begin{cases}\left(y_{1}(1+2 r(x))+r, y_{2}\right) & \text { for } y \in Y_{\mathrm{F}}^{1} \\ \left(y_{1}(1-2 r(x))+r, y_{2}\right) & \text { for } y \in Y_{\mathrm{F}}^{2}\end{cases}
$$

which then implies that the Jacobian matrix $J^{x}(y)$ is diagonal and equal to

$$
J^{x}(y)= \begin{cases}\operatorname{diag}(1+2 r(x), 1) & \text { for } y \in Y_{\mathrm{F}}^{1} \\ \operatorname{diag}(1-2 r(x), 1) & \text { for } y \in Y_{\mathrm{F}}^{2}\end{cases}
$$

Using (13) we obtain

$$
\begin{aligned}
a(\mathbf{u}, \mathbf{v} ; x)= & \frac{1}{1+2 r(x)} \int_{Y_{\mathrm{F}}^{1}} \frac{\mathbf{u}}{\partial y_{1}} \cdot \frac{\mathbf{v}}{\partial y_{1}} \mathrm{~d} y+\int_{Y_{\mathrm{F}}^{1}} \frac{\mathbf{u}}{\partial y_{2}} \cdot \frac{\mathbf{v}}{\partial y_{2}} \mathrm{~d} y \\
& +\frac{1}{1-2 r(x)} \int_{Y_{\mathrm{F}}^{2}} \frac{\mathbf{u}}{\partial y_{1}} \cdot \frac{\mathbf{v}}{\partial y_{1}} \mathrm{~d} y+\int_{Y_{\mathrm{F}}^{2}} \frac{\mathbf{u}}{\partial y_{2}} \cdot \frac{\mathbf{v}}{\partial y_{2}} \mathrm{~d} y
\end{aligned}
$$

and we get similar affine decompositions of the other two bilinear forms $(b$ and $c)$. One can regroup these terms of $A(\mathbf{U}, \mathbf{V} ; x)$ in a way that $Q_{A}=4$ and $Q_{G}=2$ with

$$
\begin{array}{cl}
\Theta_{1}^{A}(x)=\frac{1}{1+2 r(x)}, & \Theta_{2}^{A}(x)=\frac{1}{1-2 r(x)}, \quad \Theta_{3}^{A}(x)=1, \quad \Theta_{4}^{A}(x)=r(x), \\
\Theta_{1}^{G}(x)=1, \quad \Theta_{2}^{G}(x)=r(x) .
\end{array}
$$

RB space. The RB space $X_{i}$ is spanned by solutions $\mathbf{U}_{h}^{i, x}$ to (18) for a carefully selected set of parameters $S^{i}=\left\{x^{i, 1}, x^{i, 2}, \ldots, x^{i, N_{i}}\right\} \subset \Omega$, where $N_{i} \in \mathbb{N}$. Let us denote $\left\{\mathbf{U}_{h}^{i, 1}, \mathbf{U}_{h}^{i, 2}, \ldots, \mathbf{U}^{i, N_{i}}\right\}$ the result of applying the Gram-Schmidt orthogonalization procedure on $\left\{\mathbf{U}_{h}^{i, x^{i, 1}}, \mathbf{U}_{h}^{i, x^{i, 2}}, \ldots, \mathbf{U}_{h}^{i, x^{i, N_{i}}}\right\}$. We than have

$$
X_{i}=\operatorname{span}\left\{\mathbf{U}_{h}^{i, 1}, \mathbf{U}_{h}^{i, 2}, \ldots, \mathbf{U}^{i, N_{i}}\right\} .
$$

The set $S^{i}$ is constructed in the offline phase for every $i \in\{1, \ldots, d\}$. However, we first consider the online phase. 


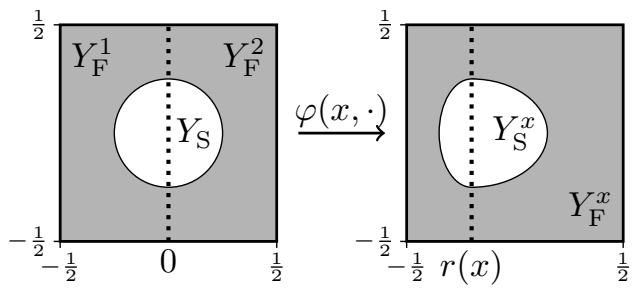

Figure 2: An illustration of the mapping $\varphi$ for the example of affine decomposition.

Online phase: evaluation of $a^{\mathrm{RB}}$. Let us consider the reduced system (20) for any $x \in \Omega$ and $i \in\{1, \ldots, d\}$ and let us look for the solution $\mathbf{U}_{\mathrm{RB}}^{i, x} \in X_{i}$ in the form $\mathbf{U}_{\mathrm{RB}}^{i, x}=$ $\sum_{n=1}^{N_{i}} \alpha_{n}^{i, x} \mathbf{U}_{h}^{i, n}$, where $\alpha^{i, x}=\left(\alpha_{1}^{i, x}, \ldots, \alpha_{N_{i}}^{i, x}\right)^{T} \in \mathbb{R}^{N_{i}}$ is a vector of unknowns. Inserting this representation into (20) and using the affine decomposition (23), (24) we arrive at a reduced system

$$
\mathbf{A}^{i, x} \alpha^{i, x}=\mathbf{G}^{i, x}
$$

where the matrix $\mathbf{A}^{i, x} \in \mathbb{R}^{N_{i} \times N_{i}}$ and the right hand side $\mathbf{G}^{i, x} \in \mathbb{R}^{N_{i}}$ are defined entry-wise by

$$
\begin{aligned}
\left(\mathbf{A}^{i, x}\right)_{n m} & =\sum_{q, r=1}^{Q_{A}} \Theta_{q}^{A}(x) \Theta_{r}^{A}(x) \underline{A^{q}\left(\mathbf{U}_{h}^{i, n}, T^{r}\left(\mathbf{U}_{h}^{i, m}\right)\right)}, \\
\left(\mathbf{G}^{i, x}\right)_{n} & =\sum_{q=1}^{Q_{A}} \sum_{r=1}^{Q_{G}} \Theta_{q}^{A}(x) \Theta_{r}^{G}(x) \underline{G^{i r}\left(T^{q}\left(\mathbf{U}_{h}^{i, n}\right)\right)} .
\end{aligned}
$$

As the underlined quantities in (27) are precomputed in the offline phase, the assembling of (27) and the solution of (26) have a time cost independent of $\operatorname{dim}\left(X_{h}\right)$. Indeed, the assembling of (27) takes $\mathcal{O}\left(N_{i}^{2} Q_{A}^{2}+N_{i} Q_{A} Q_{G}\right)$ operations. The linear system (26) is dense but of small size $N_{i}$, therefore, a direct solution takes $\mathcal{O}\left(N_{i}^{3}\right)$ operations. Using the definition of $a^{\mathrm{RB}}(x)$ in (22) and the affine decomposition (23) we get

$$
\begin{gathered}
a_{i j}^{\mathrm{RB}}(x)=\sum_{q=1}^{Q_{G}} \Theta_{q}^{G}(x)\left(\sum_{m=1}^{N_{j}} \alpha_{m}^{j, x} \underline{G^{i q}\left(\mathbf{U}_{h}^{j, m}\right)}+\sum_{n=1}^{N_{i}} \alpha_{n}^{i, x} \underline{G^{j q}\left(\mathbf{U}_{h}^{i, n}\right)}\right) \\
-\sum_{q=1}^{Q_{A}} \sum_{n=1}^{N_{i}} \sum_{m=1}^{N_{j}} \alpha_{n}^{i, x} \alpha_{m}^{j, x} \Theta_{q}^{A}(x) \underline{A^{q}\left(\mathbf{U}_{h}^{i, n}, \mathbf{U}_{h}^{i, m}\right)} .
\end{gathered}
$$

Again, by precomputing the underlined expressions in the offline phase, the evaluation of $a^{\mathrm{RB}}(x)$ is independent of $\operatorname{dim}\left(X_{h}\right)$.

Offline phase: Greedy construction of the RB space. We sketch here how the parameters $S^{i}=\left\{x^{i, 1}, \ldots, x^{i, N_{i}}\right\}$, which then define the $\mathrm{RB}$ space $X_{i}$, are selected using a greedy procedure. For details see $[4,5]$. The main ingredient is the following a posteriori error estimator. Given a RB space $X_{i}$, we can show that

$$
\left\|\mathbf{U}_{h}^{i, x}-\mathbf{U}_{\mathrm{RB}}^{i, x}\right\|_{X} \leq \Delta_{i}^{\mathrm{E}}(x):=\frac{\left\|R^{i}(\cdot ; x)\right\|_{X_{h}^{\prime}}}{\beta_{\mathrm{low}}(x)},
$$

where $R^{i}(\mathbf{V} ; x)=G^{i}(\mathbf{V} ; x)-A\left(\mathbf{U}_{\mathrm{RB}}^{i, x}, \mathbf{V} ; x\right)$ and $\beta_{\text {low }}(x)$ is some positive lower bound of the inf-sup stability constant $\beta(x)$ of $A(\cdot, \cdot ; x)$. A great advantage of the a posteriori error estimator $\Delta_{i}^{\mathrm{E}}(x)$ is that it can be evaluated cheaply for any $x \in \Omega$ thanks to an appropriate offline precomputation. Indeed, we can define $\beta_{\text {low }}(x)$ using the successive constraint method [17] (see below) or some of its approximations mentioned in [5]. The residual term $\left\|R^{i}(\cdot ; x)\right\|_{X_{h}^{\prime}}$ enjoys a similar offline/online decomposition as the effective permeability $a^{\mathrm{RB}}$. We can now define the greedy algorithm.

Algorithm: Greedy RB construction. Select a training set of parameters $\Xi_{\text {train }} \subset \Omega$ and an RB tolerance $\varepsilon_{\text {tol }}$. For each $i \in\{1, \ldots, d\}$ we start with $S^{i}=\emptyset$ and repeat:

1. Find $\hat{x} \in \Xi_{\text {train }}$ for which the value $\Delta_{i}^{\mathrm{E}}(\hat{x})$ is the largest.

2. If $\Delta_{i}^{\mathrm{E}}(\hat{x})$ is lower $\varepsilon_{\mathrm{tol}}$, we stop the algorithm. Else, we add $\hat{x}$ to $S^{i}$, update the space $X_{i}$, compute all the offline coefficients necessary for evaluation of the a posteriori error estimator, and continue with step 1. 
Successive constraint method. For any $x \in \Omega$ the inf-sup constant $\beta(x)$ can be interpreted as $\sqrt{\lambda_{\min }}$, where $\lambda_{\min }$ is a minimal eigenvalue of a generalized eigenvalue problem of the type $A z=\lambda B z$ with $A$ and $B$ symmetric and positive definite. However, solving this eigenproblem numerically for every $x \in \Xi_{\text {train }}$ can be prohibitive. The successive constraint method [17] is a greedy offline-online algorithm that computes $\beta(\bar{x})$ exactly for a small number of parameters $\bar{x} \in S \subset \Omega$ and then uses a rigorous bound $\beta(x) \geq \beta_{\text {low }}(x):=$ $\max _{\bar{x} \in S} \beta(\bar{x}) \beta_{\mathrm{LB}}(x ; \bar{x})$, where the online computation of the term $\beta_{\mathrm{LB}}(x ; \bar{x})$ involves solving a small linear programing problem.

3.4. The fully discrete multiscale method. By combining the SIP-DG-FEM discretization (10) from section 3.1 and the reduced basis approximation of the effective permeability from section 3.2 we obtain the fully discrete multiscale method: Find $p^{H, \mathrm{RB}} \in S_{\mathrm{dis}}^{l}\left(\mathcal{T}_{H}\right)$ such that

$$
B_{H}^{\mathrm{RB}}\left(p^{H, \mathrm{RB}}, q^{H}\right)=L_{H}^{\mathrm{RB}}\left(q^{H}\right) \quad \forall q^{H} \in S_{\mathrm{dis}}^{l}\left(\mathcal{T}_{H}\right),
$$

where

$$
\begin{aligned}
B_{H}^{\mathrm{RB}}\left(p^{H}, q^{H}\right)= & \int_{\Omega} \Pi_{a^{\mathrm{RB}}}\left(\nabla p^{H}\right) \cdot \nabla q^{H} \mathrm{~d} x-\sum_{e \in \mathcal{E}_{\mathrm{pen}}} \int_{e}\left(\left\{\Pi_{a^{\mathrm{RB}}}\left(\nabla p^{H}\right)\right\} \cdot \llbracket q^{H} \rrbracket\right. \\
& \left.+\left\{\Pi_{a^{\mathrm{RB}}}\left(\nabla q^{H}\right)\right\} \cdot \llbracket p^{H} \rrbracket-\sigma \llbracket p^{H} \rrbracket \cdot \llbracket q^{H} \rrbracket\right) \mathrm{d} s, \\
L_{H}^{\mathrm{RB}}\left(q^{H}\right)= & \int_{\Omega} f q^{H} \mathrm{~d} x+\int_{\Gamma_{\mathrm{D}}}\left(\Pi_{a^{\mathrm{RB}}}\left(\nabla q^{H}\right) \cdot \mathbf{n}+\sigma q^{H}\right) g_{\mathrm{D}} \mathrm{d} s+\int_{\Gamma_{\mathrm{N}}} g_{\mathrm{N}} q^{H} \mathrm{~d} s .
\end{aligned}
$$

For simplicity, we assume that the boundary data $g_{\mathrm{D}}$ and $g_{\mathrm{N}}$ can be integrated exactly in (30).

\section{Analysis of the method}

In this section we study the stability and the convergence of the multiscale method (29). Accuracy of the approximation of $a^{0}$ by $a^{\mathrm{RB}}$ is studied in section 4.1, well-posedness in section 4.2, and a priori error estimates in section 4.3.

Spaces and norms. While the numerical multiscale method is defined on $S_{\text {dis }}^{l}\left(\mathcal{T}_{H}\right)$, an appropriate space for the analysis is

$$
V\left(\mathcal{T}_{H}\right)=S_{\text {dis }}^{l}\left(\mathcal{T}_{H}\right)+H_{0}^{1}(\Omega) \cap H^{2}(\Omega)
$$

accompanied with a mesh-dependent norm

$$
\|v\|=\left(\|v\|_{0}^{2}+\sum_{K \in \mathcal{T}_{H}} H_{K}^{2}|v|_{2, K}^{2}\right)^{1 / 2}
$$

where

$$
\|v\|_{0}^{2}=\|\nabla v\|_{L^{2}(\Omega)}^{2}+|v|_{*}^{2}, \quad|v|_{m, K}^{2}=\sum_{|\alpha|=m}\left\|\partial^{\alpha} v\right\|_{L^{2}(K)}^{2}, \quad|v|_{*}^{2}=\sum_{e \in \mathcal{E}_{\mathrm{pen}}}\left\|H_{e}^{-1 / 2} \llbracket v \rrbracket\right\|_{L^{2}(e)}^{2} .
$$

Both $\|\cdot\|$ and $\|\cdot\|_{0}$ are norms in $V\left(\mathcal{T}_{H}\right)$ but they are not equivalent. However, using the local inverse inequality it can be shown that they are equivalent when restricted to $S_{\mathrm{dis}}^{l}\left(\mathcal{T}_{H}\right)$.

Trace inverse inequality. For any $K \in \mathcal{T}_{H}$ and vector function $\mathbf{v} \in \mathcal{P}^{l-1}(K)^{d}$ we have

$$
\|\mathbf{v}\|_{L^{2}(\partial K)} \leq C l H_{K}^{-1 / 2}\|\mathbf{v}\|_{L^{2}(K)}
$$

where $C$ depends only on $d$ and shape-regularity of $K$. For a proof see [25].

4.1. Properties of the effective permeability. In [22], where periodic porous media are studied, it is shown that $a^{0}(x)$ is symmetric and coercive for any given $x \in \Omega$. In our analysis we will require a uniform coercivity and boundedness constant for non-periodic medium. It is shown in [3] that under rather generic assumptions on the varying micro geometries $\left(Y_{\mathrm{F}}^{x}, Y_{\mathrm{S}}^{x}\right)$ there exist $\Lambda \geq \lambda>0$ such that

$$
a^{0}(x) \xi \cdot \xi \geq \lambda|\xi|^{2}, \quad\left\|a^{0}(x)\right\|_{\mathrm{F}} \leq \Lambda \quad \forall x \in \Omega, \quad \forall \xi \in \mathbb{R}^{d} .
$$

For any macroscopic mesh $\mathcal{T}_{H}$ the tensors $a^{h}(x)$ and $a^{\mathrm{RB}}(x)$ are considered (in the multiscale methods) only on the quadrature points $Q_{H}=\left\{x_{K_{j}}: K \in \mathcal{T}_{H}, j \in\{1, \ldots, J\}\right\}$. Assuming 
that the micro mesh size $h$ is small enough, bounds of type (34) are shown for $a^{h}(x)$ in [3]. Assuming that $\varepsilon_{\text {tol }}$ in the offline RB process is small enough and the training set $\Xi_{\text {train }}$ is dense enough in $\Omega$, bounds of type (34) can be obtained for $a_{\mathrm{RB}}(x)$ too (see [5]). Thus, for the rest of the analysis we assume that

$$
a^{\mathrm{RB}}(x) \xi \cdot \xi \geq \lambda|\xi|^{2}, \quad\left\|a^{\mathrm{RB}}(x)\right\|_{\mathrm{F}} \leq \Lambda \quad \forall x \in Q_{H}, \quad \forall \xi \in \mathbb{R}^{d} .
$$

If we know $Q_{H}$ while running the offline phase, we can ensure (35) by having $Q_{H} \subset \Xi_{\text {train }}$.

Permeability approximation error. We decompose the difference $a^{0}-a^{\mathrm{RB}}$ into two terms: $a^{0}-a^{h}$ and $a^{h}-a^{\mathrm{RB}}$. It can be shown that (see [3])

$$
\left\|a^{0}(x)-a^{h}(x)\right\|_{\mathrm{F}}^{2} \leq \sum_{i=1}^{d}\left\|\mathbf{u}^{i, x}(\cdot)-\mathbf{u}^{i, x, h}(\phi(x, \cdot))\right\|_{L^{2}\left(Y_{\mathrm{F}}\right)}^{2} .
$$

Using standard a priori error estimates on the Stokes micro problems, we have $\| a^{0}(x)-$ $a^{h}(x) \|_{\mathrm{F}} \leq C h^{k+2}$, if $\mathbf{u}^{i, x} \in H^{k+1}\left(Y_{\mathrm{F}}^{x}\right)$ and the Taylor-Hood $\mathcal{P}^{k+1} / \mathcal{P}^{k}$ finite elements are used on the micro scale. Expressed in terms of microscopic degrees of freedom $N_{\text {mic }}$, this error estimate reads

$$
\left\|a^{0}(x)-a^{h}(x)\right\|_{\mathrm{F}} \leq C N_{\text {mic }}^{\frac{-k-2}{d}}
$$

The required regularity of $\mathbf{u}^{i, x}$ can be difficult to achieve, since the micro domains usually contain re-entrant corners. Hence, as shown in [3], adaptive mesh refinement must be used to obtain such convergence rates. The error caused by the reduced basis approximation can be bounded by (see [5])

$$
\left\|a^{h}(x)-a^{\mathrm{RB}}(x)\right\|_{\mathrm{F}} \leq C \sum_{i=1}^{d} \inf _{V \in X_{i}}\left\|\mathbf{U}_{h}^{i, x}-V\right\|_{X}^{2} \quad \forall x \in \Omega
$$

To estimate the projection error of the solutions $\mathbf{U}_{h}^{i, x}$ with respect to the low-dimensional space $X_{i}$ we rely on some properties of the solution manifolds $\mathcal{M}_{i}=\left\{\mathbf{U}_{h}^{i, x} ; x \in \Omega\right\}$. For any $\mathcal{M} \subset X_{h}$ we define the Kolmogorov $n$-width

$$
d_{n}(\mathcal{M})=\inf _{\substack{Z \in X_{h} \\ \operatorname{dim}(Z)=n}} \sup _{\mathbf{U} \in \mathcal{M}} \inf _{\mathbf{V} \in Z}\|\mathbf{V}-\mathbf{U}\|_{X}
$$

If there are constants $M, a, \alpha>0$ such that $d_{n}\left(\mathcal{M}_{i}\right) \leq M e^{-a n^{\alpha}}$ for every $i \in\{1, \ldots, d\}$, then we can show that

$$
\left\|a^{h}(x)-a^{\mathrm{RB}}(x)\right\|_{\mathrm{F}} \leq C M e^{-c N_{\mathrm{RB}}^{\beta}},
$$

where $C, c>0$ and $\beta=\alpha /(\alpha+1)$ and $N_{\mathrm{RB}}=\min _{i \in\{1, \ldots, d\}} N_{i}$.

4.2. Stability. Stability of the multiscale method (29) is closely related to the penalty weight $\sigma: \cup_{e \in \mathcal{E}_{\text {pen }}} e \rightarrow \mathbb{R}$. For any $e \in \mathcal{E}$ we let $\sigma$ be constant in $e$ by defining $\left.\sigma\right|_{e} \equiv \alpha S_{e} / H_{e}$, where $H_{e}=\operatorname{diam}(e), \alpha>0$ is a large enough global constant (see Theorem 3 ), and

$$
S_{e} \equiv \max _{\substack{K \in \mathcal{T}_{H} \\ e \subset \partial K}} S_{K}, \quad S_{K}=\max _{j \in\{1, \ldots, J\}}\left\|a^{\mathrm{RB}}\left(x_{K_{j}}\right)\right\|_{F} \quad \forall K \in \mathcal{T}_{H} .
$$

The explicit inclusion of $S_{e}$ allows us to find stability bounds for $\alpha$ that are independent of the tensor scaling (see Remark 4). Notice also that we have $S_{e} \leq S_{K} \leq \Lambda$ for every $K \in \mathcal{T}_{H}$ and $e \in \partial K$. We continue by two lemmas that consider an arbitrary tensor defined on all quadrature points $Q_{H}$. These lemmas generalize results in [2] for higher order macro polynomials.

Lemma 1. Consider a tensor a $: Q_{H} \rightarrow \mathbb{R}^{d \times d}$ such that $\|a(x)\|_{\mathrm{F}} \leq \Lambda$ for every $x \in Q_{H}$. If (Q) and (35) hold, then for every $p^{H}, q^{H} \in S_{\mathrm{dis}}^{l}\left(\mathcal{T}_{H}\right)$ and $K \in \mathcal{T}_{H}$ we have

$$
\begin{gathered}
\int_{K} \Pi_{a}\left(\nabla p^{H}\right) \cdot \nabla q^{H} \mathrm{~d} x \leq \Lambda\left\|\nabla p^{H}\right\|_{L^{2}(K)}\left\|\nabla q^{H}\right\|_{L^{2}(K)}, \\
\left\|\Pi_{a}\left(\nabla p^{H}\right)\right\|_{L^{2}(K)} \leq \Lambda\left\|\nabla p^{H}\right\|_{L^{2}(K)} .
\end{gathered}
$$

If the tensor satisfies $a(x) \xi \cdot \xi \geq \lambda|\xi|^{2}>0$ for every $x \in Q^{H}$ and $\xi \in \mathbb{R}^{d}$, we have

$$
\int_{K} \Pi_{a}\left(\nabla p^{H}\right) \cdot \nabla p^{H} \mathrm{~d} x \geq \lambda\left\|\nabla p^{H}\right\|_{L^{2}(K)}^{2} .
$$


Proof. By (Q) the integral on the left side of (39) can be evaluated exactly by the quadrature formula since the integrand is a polynomial of degree $2(l-1)$. This observation and the Cauchy-Schwarz inequality give

$$
\begin{aligned}
\int_{K} \Pi_{a}\left(\nabla p^{H}\right) \cdot \nabla q^{H} \mathrm{~d} x & =\sum_{j=1}^{J} \omega_{K_{j}} a\left(x_{K_{j}}\right) \nabla p^{H}\left(x_{K_{j}}\right) \cdot \nabla q^{H}\left(x_{K_{j}}\right) \\
& \leq \Lambda_{a} \sum_{j=1}^{J} \omega_{K_{j}}\left|p^{H}\left(x_{K_{j}}\right)\right| \cdot\left|q^{H}\left(x_{K_{j}}\right)\right| \\
& \leq \Lambda_{a}\left(\sum_{j=1}^{J} \omega_{K_{j}}\left|p^{H}\left(x_{K_{j}}\right)\right|^{2}\right)^{1 / 2}\left(\sum_{j=1}^{J} \omega_{K_{j}}\left|q^{H}\left(x_{K_{j}}\right)\right|^{2}\right)^{1 / 2} \\
& \leq \Lambda_{a}\left\|\nabla p^{H}\right\|_{L^{2}(K)}\left\|\nabla q^{H}\right\|_{L^{2}(K)} .
\end{aligned}
$$

Proof of the other two bounds (40) and (41) is analogous.

Lemma 2. Consider a tensor a $: Q_{H} \rightarrow \mathbb{R}^{d \times d}$ such that $\|a(x)\|_{\mathrm{F}} \leq \Lambda$ for every $x \in Q_{H}$. If $(\boldsymbol{Q})$ holds then for any $p^{H}, q^{H} \in S_{\mathrm{dis}}^{l}\left(\mathcal{T}_{H}\right)$ we have

$$
\sum_{e \in \mathcal{E}_{\mathrm{pen}}} \int_{e}\left\{\Pi_{a}\left(\nabla p^{H}\right)\right\} \cdot \llbracket q^{H} \rrbracket \mathrm{d} s \leq C \Lambda\left\|\nabla p^{H}\right\|_{L^{2}(\Omega)}\left|q^{H}\right|_{*},
$$

where the constant $C$ is depends only on $l, d$, and the shape-regularity of $\mathcal{T}_{H}$.

Proof. The Cauchy-Schwarz inequality gives

$$
\sum_{e \in \mathcal{E}_{\mathrm{pen}}} \int_{e}\left\{\Pi_{a}\left(\nabla p^{H}\right)\right\} \cdot \llbracket q^{H} \rrbracket \mathrm{d} s \leq(\underbrace{\sum_{e \in \mathcal{E}_{\mathrm{pen}}} H_{e}\left\|\left\{\Pi_{a}\left(\nabla p^{H}\right)\right\}\right\|_{L^{2}(e)}^{2}}_{=: I})^{1 / 2}\left|q^{H}\right|_{*} .
$$

For any $e \in \mathcal{E}$ we consider the neighboring elements $K_{e}^{-}, K_{e}^{+} \in \mathcal{T}_{H}$, where $K_{e}^{-}=K_{e}^{+}$for the boundary edges $e \in \partial \Omega$. Using the triangle and Cauchy's inequalities we get

$$
\left\|\left\{\Pi_{a}\left(\nabla p^{H}\right)\right\}\right\|_{L^{2}(e)}^{2} \leq \frac{1}{2}\left(\left\|\left.\Pi_{a}\left(\nabla p^{H}\right)\right|_{K_{e}^{-}}\right\|_{L^{2}(e)}^{2}+\left\|\left.\Pi_{a}\left(\nabla p^{H}\right)\right|_{K_{e}^{+}}\right\|_{L^{2}(e)}^{2}\right)
$$

where $C$ depends only on the shape-regularity of $\mathcal{T}_{H}$. Next we bound $I$ by using first that $H_{e} \leq C H_{K}$ (shape regularity) and the inequality (44), then the trace inverse inequality (33) and (40). We obtain

$$
\begin{aligned}
I & \leq C \sum_{K \in \mathcal{T}_{H}} H_{K}\left\|\left.\Pi_{a}\left(\nabla p^{H}\right)\right|_{K}\right\|_{L^{2}(\partial K)}^{2} \leq C \sum_{K \in \mathcal{T}_{H}} l^{2}\left\|\Pi_{a}\left(\nabla p^{H}\right)\right\|_{L^{2}(K)}^{2} \\
& \leq C \sum_{K \in \mathcal{T}_{H}} \Lambda^{2}\left\|\nabla p^{H}\right\|_{L^{2}(K)}^{2} \leq C \Lambda^{2}\left\|\nabla p^{H}\right\|_{L^{2}(\Omega)}^{2} .
\end{aligned}
$$

We conclude by using (45) in (43).

Theorem 3. Assume that (35) and $(\boldsymbol{Q})$ hold. Then there is a threshold value $\alpha_{\min }>0$ such that for $\alpha>\alpha_{\min }$ the bilinear form $B_{H}^{\mathrm{RB}}(\cdot, \cdot)$ (see (30)) is uniformly elliptic and bounded on $S_{\mathrm{dis}}^{l}\left(\mathcal{T}_{H}\right) \times S_{\mathrm{dis}}^{l}\left(\mathcal{T}_{H}\right)$ and the problem $(29)$ has a unique solution $p^{H, \mathrm{RB}} \in S_{\mathrm{dis}}^{l}\left(\mathcal{T}_{H}\right)$.

Proof. We recall that the penalty parameter $\alpha$ appears in the penalty weight $\sigma$ and we will show that for $\alpha$ large enough the bilinear form $B_{H}^{\mathrm{RB}}(\cdot, \cdot)$ is coercive and bounded. The result will then follow from the Lax-Milgram lemma.

Coercivity. For any $p^{H} \in S_{\mathrm{dis}}^{l}\left(\mathcal{T}_{H}\right)$ we consider $B_{H}^{\mathrm{RB}}\left(p^{H}, p^{H}\right)$ that we split as in (30) into three terms. We then obtain a lower bound by using (41), Lemma 2 with $a=a^{\mathrm{RB}}$, and (35)

$$
\begin{aligned}
& B_{H}^{\mathrm{RB}}\left(p^{H}, p^{H}\right)= \int_{\Omega} \Pi_{a^{\mathrm{RB}}}\left(\nabla p^{H}\right) \cdot \nabla p^{H} \mathrm{~d} x-2 \sum_{e \in \mathcal{E}_{\mathrm{pen}}} \int_{e}\left\{\Pi_{a^{\mathrm{RB}}}\left(\nabla p^{H}\right)\right\} \cdot \llbracket p^{H} \rrbracket \mathrm{d} s \\
&+\sum_{e \in \mathcal{E}_{\text {pen }}} \int_{e} \sigma \llbracket p^{H} \rrbracket \cdot \llbracket p^{H} \rrbracket \mathrm{d} s \\
& \geq \lambda\left\|\nabla p^{H}\right\|_{L^{2}(\Omega)}^{2}-C \Lambda\left\|\nabla p^{H}\right\|_{L^{2}(\Omega)}\left|p^{H}\right|_{*}+\alpha \lambda\left|p_{H}\right|_{*}^{2} .
\end{aligned}
$$


Using the inequality $2 x y \leq \alpha^{-1 / 2} x^{2}+\alpha^{1 / 2} y^{2}$ on the mixed term and then assuming that $\alpha>\max \left\{1,4 C^{2} \Lambda^{2} \lambda^{-2}\right\}$ we get

$$
\begin{aligned}
B_{H}^{\mathrm{RB}}\left(p^{H}, p^{H}\right) & \geq\left(\lambda-C \Lambda \alpha^{-1 / 2}\right)\left\|\nabla p^{H}\right\|_{L^{2}(\Omega)}^{2}+\left(\alpha \lambda-C \Lambda \alpha^{1 / 2}\right)\left|p_{H}\right|_{*}^{2} \\
& \geq \frac{\lambda}{2}\left(\left\|\nabla p^{H}\right\|_{L^{2}(\Omega)}^{2}+\left|p_{H}\right|_{*}^{2}\right)=\frac{\lambda}{2}\left\|p^{H}\right\|_{0}^{2} \geq C\left\|p^{H}\right\|,
\end{aligned}
$$

where the last constant $C$ depends only on $\lambda, d, l$, and the shape-regularity of $\mathcal{T}_{H}$.

Boundedness. For any $p^{H}, q^{H} \in S_{\mathrm{dis}}^{l}\left(\mathcal{T}_{H}\right)$ we can bound $\left|B_{H}^{\mathrm{RB}}\left(p^{H}, q^{H}\right)\right|$ from above by using (39) and Lemma 2 with $a=a^{\mathrm{RB}}$, using that $S_{K}, S_{e} \leq \Lambda$ and the Cauchy-Schwarz inequality. We get

$$
\begin{aligned}
\left|B_{H}^{\mathrm{RB}}\left(p^{H}, q^{H}\right)\right| \leq & \Lambda\left\|p^{H}\right\|_{L^{2}(\Omega)}\left\|q^{H}\right\|_{L^{2}(\Omega)}+C \Lambda\left\|\nabla p^{H}\right\|_{L^{2}(\Omega)}\left|q^{H}\right|_{*}+C \Lambda\left\|\nabla q^{H}\right\|_{L^{2}(\Omega)}\left|p^{H}\right|_{*} \\
& +\alpha \sum_{e \in \mathcal{E}_{\mathrm{pen}}} S_{e}\left\|H_{e}^{-1 / 2} \llbracket p^{H} \rrbracket\right\|_{L^{2}(e)}\left\|H_{e}^{-1 / 2} \llbracket q^{H} \rrbracket\right\|_{L^{2}(e)} \\
\leq & C\left(\left\|p^{H}\right\|_{L^{2}(\Omega)}+\left|p^{H}\right|_{*}\right)\left(\left\|\nabla q^{H}\right\|_{L^{2}(\Omega)}+\left|q^{H}\right|_{*}\right) \\
\leq & C\left\|p^{H}\right\|_{0}\left\|q^{H}\right\|_{0} \leq C\left\|p^{H}\right\|\|\| q^{H} \|
\end{aligned}
$$

where $C$ depends on $\Lambda, \alpha, d, l$, and the shape-regularity of $\mathcal{T}_{H}$.

Remark 4. If the proof of coercivity in Theorem 3 is carried a bit differently and one follows the constants, one can obtain a better condition on $\alpha$, for example

$$
\alpha \geq 4 C^{2} l^{2} \max _{K \in \mathcal{T}_{H}} S_{K}^{2} / s_{K}^{2},
$$

where the constant $C$ depends only on $d$ and shape-regularity of $\mathcal{T}_{H}$ and $s_{K}>0$ is such that $a^{\mathrm{RB}}\left(x_{K_{j}}\right) \xi \cdot \xi \geq s_{K}|\xi|^{2}$ is valid for every $j \in\{1, \ldots, J\}$. In practice, setting $\alpha=10 l^{2}$ gives good results.

4.3. A priori error estimates. We decompose the error into two parts (and estimate them independently)

$$
\left\|p^{0}-p^{H, \mathrm{RB}}\right\| \leq\|\underbrace{p^{0}-p^{H, 0}}_{e_{\text {mac }}}\| \mid+\|\underbrace{p^{H, 0}-p^{H, \mathrm{RB}}}_{e_{\text {mic }, \mathrm{RB}}}\|
$$

Micro and RB error. The problems (10) and (29) differ only in the tensor values at the quadrature points of $\mathcal{T}_{H}$. Therefore, following the proof of Theorem 3, we can equally prove that the problem (10) is well-posed for $\alpha$ large enough. That is, there exists a unique solution $p^{H, 0} \in S_{\text {dis }}^{l}\left(\mathcal{T}_{H}\right)$ for $\alpha$ large enough.

Lemma 5. We have

$$
\left\|e_{\text {mic, } \mathrm{RB}}\right\| \leq C(\underbrace{\max _{x \in Q_{H}}\left\|a^{\mathrm{RB}}(x)-a^{0}(x)\right\|_{\mathrm{F}}}_{C_{a}})\left(\left\|p^{H, \mathrm{RB}}\right\|+\left\|g_{\mathrm{D}}\right\|_{H^{1 / 2}\left(\Gamma_{\mathrm{D}}\right)}\right) .
$$

Proof. Coercivity of $B_{H}^{0}(\cdot, \cdot)$ gives

$$
\begin{aligned}
C\left\|e_{\mathrm{mic}, \mathrm{RB}}\right\|^{2} & \leq B_{H}^{0}\left(e_{\mathrm{mic}, \mathrm{RB}}, e_{\mathrm{mic}, \mathrm{RB}}\right) \\
& =B_{H}^{0}\left(p^{H, 0}, e_{\mathrm{mic}, \mathrm{RB}}\right)-B_{H}^{0}\left(p^{H, \mathrm{RB}}, e_{\mathrm{mic}, \mathrm{RB}}\right) \\
& =B_{H}^{\mathrm{RB}}\left(p^{H, \mathrm{RB}}, e_{\mathrm{mic}, \mathrm{RB}}\right)-B_{H}^{0}\left(p^{H, \mathrm{RB}}, e_{\text {mic }, \mathrm{RB}}\right)+L_{H}^{0}\left(e_{\mathrm{mic}, \mathrm{RB}}\right)-L_{H}^{\mathrm{RB}}\left(e_{\mathrm{mic}, \mathrm{RB}}\right) .
\end{aligned}
$$

That implies

$$
\left\|e_{\mathrm{mic}, \mathrm{RB}}\right\| \leq C \sup _{q^{H} \in S_{\mathrm{dis}}^{l}\left(\mathcal{T}_{H}\right)} \frac{B_{H}^{\mathrm{RB}}\left(p^{H, \mathrm{RB}}, q^{H}\right)-B_{H}^{0}\left(p^{H, \mathrm{RB}}, q^{H}\right)+L_{H}^{0}\left(q^{H}\right)-L_{H}^{\mathrm{RB}}\left(q^{H}\right)}{\left\|q^{H}\right\|} .
$$

For any $p^{H}, q^{H} \in S_{\text {dis }}^{l}\left(\mathcal{T}_{H}\right)$ we use (39) and Lemma (2) with $a=a^{\mathrm{RB}}-a^{0}$ and then CauchySchwarz inequality to get

$$
\begin{aligned}
B_{H}^{\mathrm{RB}}( & \left.p^{H}, q^{H}\right)-B_{H}^{0}\left(p^{H}, q^{H}\right)=\int_{\Omega} \Pi_{\left(a^{\mathrm{RB}}-a^{0}\right)}\left(\nabla p^{H}\right) \cdot \nabla q^{H} \mathrm{~d} x \\
& -\sum_{e \in \mathcal{E}_{\mathrm{pen}}} \int_{e}\left(\left\{\Pi_{\left(a^{\mathrm{RB}}-a^{0}\right)}\left(\nabla p^{H}\right)\right\} \cdot \llbracket q^{H} \rrbracket+\left\{\Pi_{\left(a^{\mathrm{RB}}-a^{0}\right)}\left(\nabla q^{H}\right)\right\} \cdot \llbracket p^{H} \rrbracket\right) \mathrm{d} s \\
\leq & C C_{a}\left(\left\|\nabla p^{H}\right\|_{L^{2}(\Omega)}\left\|\nabla q^{H}\right\|_{L^{2}(\Omega)}+\left\|\nabla p^{H}\right\|_{L^{2}(\Omega)}\left|q^{H}\right|_{*}+\left|p^{H}\right|_{*}\left\|\nabla q^{H}\right\|_{L^{2}(\Omega)}\right) \\
\leq & C C_{a}\left\|p^{H}\right\|\|\| q^{H} \| .
\end{aligned}
$$


Similarly, we have

$$
\begin{aligned}
L_{H}^{0}\left(q^{H}\right)-L_{H}^{\mathrm{RB}}\left(q^{H}\right) & =\sum_{e \in \mathcal{E}_{\mathrm{D}}} \int_{e} \Pi_{\left(a^{0}-a^{\mathrm{RB}}\right)}\left(\nabla q^{H}\right) \cdot \mathbf{n} g_{\mathrm{D}} \mathrm{d} s \\
& \leq\left\|\Pi_{\left(a^{0}-a^{\mathrm{RB}}\right)}\left(\nabla q^{H}\right)\right\|_{H^{-1 / 2}\left(\Gamma_{\mathrm{D}}\right)}\left\|g_{\mathrm{D}}\right\|_{H^{1 / 2}\left(\Gamma_{\mathrm{D}}\right)} \\
& \leq C\left\|\Pi_{\left(a^{0}-a^{\mathrm{RB}}\right)}\left(\nabla q^{H}\right)\right\|_{L^{2}(\Omega)}\left\|g_{\mathrm{D}}\right\|_{H^{1 / 2}\left(\Gamma_{\mathrm{D}}\right)} \\
& \leq C C_{a}\left\|q_{H}\right\|\left\|g_{\mathrm{D}}\right\|_{H^{1 / 2}\left(\Gamma_{\mathrm{D}}\right)} .
\end{aligned}
$$

Combining the last three inequalities we get the desired result.

Macro error. We now consider the macro error $e_{\text {mac }}=p^{0}-p^{H, 0}$, which can be seen as the error of a single-scale DG method with numerical quadrature. It is standard in the DG literature to assume an additional condition on $a^{0}$ that we have not introduced yet, since we were able to carry the results in general.

Lemma 6. If $a^{0}(x)$ is constant in each element $K \in \mathcal{T}_{H}$ and $p^{0} \in H^{l+1}(\Omega)$, then

$$
\left\|p^{0}-p^{H, 0}\right\| \leq C H^{l}, \quad\left\|p^{0}-p^{H, 0}\right\|_{L^{2}(\Omega)} \leq C H^{l+1},
$$

where the constant $C$ is independent of $H$.

Proof. This is a standard result, see [11].

Theorem 7. If $a^{0}(x)$ is constant in each element $K \in \mathcal{T}_{H}$ and $p^{0} \in H^{l+1}(\Omega)$, then

$$
\left\|p^{0}-p^{H, \mathrm{RB}}\right\| \leq \leq C\left(H^{l}+\max _{x \in Q^{H}}\left\|a^{\mathrm{RB}}(x)-a^{0}(x)\right\|_{\mathrm{F}}\right)
$$

Proof. It follows from the error decomposition (48) and from Lemma 6 and Lemma 5.

We remind that under suitable assumptions on the regularity of micro problems and on the Kolmogorov $n$-width of the solution manifold we had the micro and RB estimates (36) and (38), respectively. Writing everything in the terms of degrees of freedom we obtain an error estimate

$$
\left\|p^{0}-p^{H, \mathrm{RB}}\right\| \leq C\left(N_{\mathrm{mac}}^{-\frac{l}{d}}+N_{\mathrm{mic}}^{\frac{-k-2}{d}}+e^{-c N_{\mathrm{RB}}^{\beta}}\right) .
$$

\section{$5 \quad$ Numerical experiments}

In this section we validate the proposed DG multiscale method, study convergence rates and conservative properties. We illustrate the efficiency of the method on 2D and 3D problems.

Implementation. All experiments were performed on a single computer with two 8-core processors Intel Xeon E5-2600 and 64 GB of RAM with Matlab R2014a. The finite element code is inspired by $[8,15]$ and it uses vectorization techniques to achieve fast assembling. Sparse linear systems are solved by the Matlab routine mldivide for two-dimensional problems. For three-dimensional problems we apply two different techniques.

- Positive definite systems are solved by the algebraic multigrid solver AGMG [18].

- Stokes systems are solved by the Uzawa method [19]. In the Uzawa method, AGMG was used as a preconditioner for the coercive part and the diagonal of the pressure mass matrix was used as a preconditioner of the Shur's complement.

Linear systems with the same positive definite matrix representing the scalar product on $X_{h}$ are solved repeatedly in the offline algorithms. We optimize this by precomputing a sparse Cholesky factorization (Matlab routine chol). Generalized eigenproblems from the SCM method were solved using the Matlab package bleigifp [21], which implements a block, inverse-free Krylov subspace method. Linear programming problems from the SCM method are solved by the Matlab routine linprog with the default settings. 
5.1. A 2D problem. Let $\Omega=(-3,3) \times(-2,2)$ and define the local porous geometries $\left(Y_{\mathrm{F}}^{x}, Y_{\mathrm{S}}^{x}\right)$ by (see Figure 3$)$

$$
Y_{\mathrm{F}}^{x}=\left\{y \in Y:\left|y_{1}\right|<a \text { or }\left|y_{2}\right|<c \text { or } \frac{\left(\left|y_{1}\right|-a\right)^{2}}{(b-a)^{2}}+\frac{\left(\left|y_{2}\right|-c\right)^{2}}{(d-c)^{2}}<1\right\},
$$

where $a, b, c, d$ are functions depending on $x$ (see (55)). We define the reference porous geometry $Y_{\mathrm{F}}$ by setting $a=b=1 / 6$ and $c=d=1 / 3$. Figure 3 displays how can we divide $Y_{\mathrm{F}}$ by four horizontal and four vertical lines and how can we obtain $Y_{\mathrm{F}}^{x}$ by simply moving these lines so that the geometry is stretched or contracted in the directions $y_{1}$ and $y_{2}$. That is, we can divide $Y_{\mathrm{F}}$ into 13 regions such that an implicitly defined $\varphi(x, \cdot)$ will be affine in each region. To avoid degenerate cases we set the mapping $x \mapsto(a, b, c, d)$ so that $0<a(x)<b(x)<1 / 2$ and $0<c(x)<d(x)<1 / 2$. Let

$$
\begin{gathered}
a(x)=0.15 e(x)+0.05, \quad c(x)=0.15 f(x)+0.05, \\
b(x)=d(x)=0.15(e(x)+f(x))+0.1,
\end{gathered}
$$

where $e(x)=\sin \left(\pi x_{1} / 6+x_{2}\right)^{2}$ and $f(x)=\cos \left(\pi x_{1} / 6-x_{2}\right)^{2}$. We plot some of the extreme deformations of the reference geometry in Figure 4 . Notice that the permeability of thick and thin channels differs by two orders of magnitude.
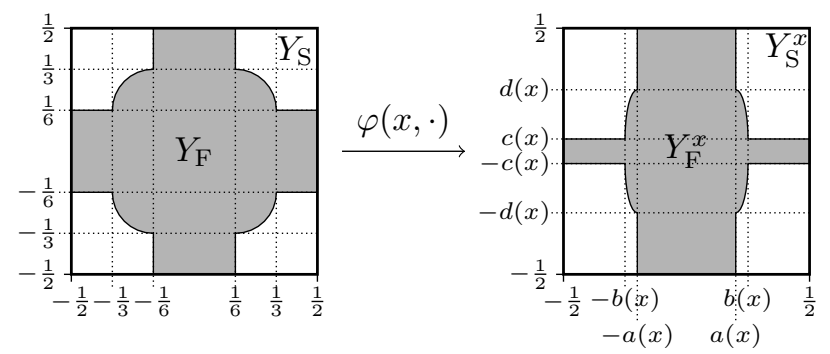

Figure 3: Micro geometries and the mapping $\varphi(x, \cdot)$ for the 2D example.
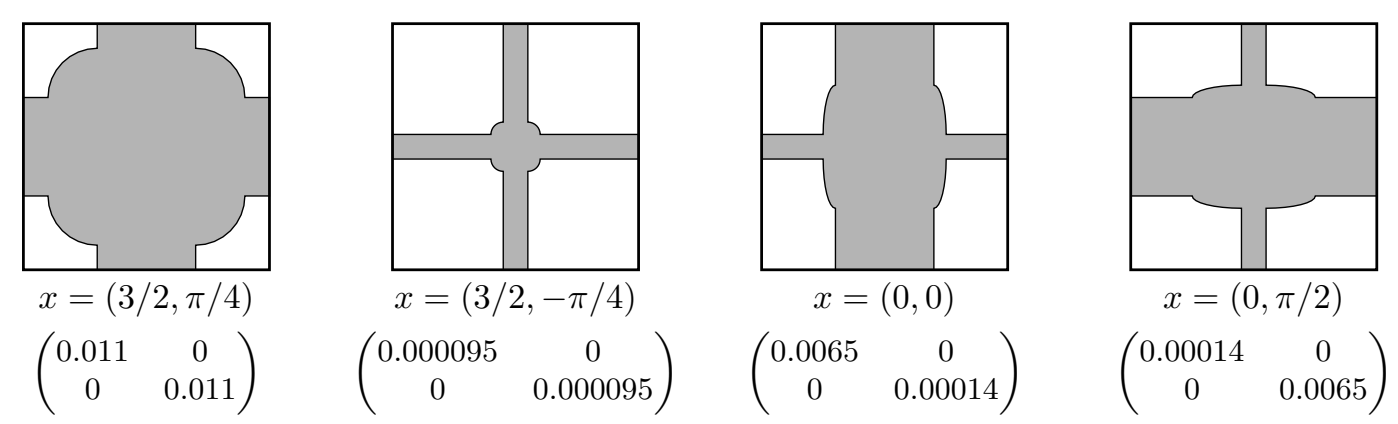

Figure 4: Examples of the local porous geometries $\left(Y_{\mathrm{F}}^{x}, Y_{\mathrm{S}}^{x}\right)$ that show extremal deformations. The matrices below are approximate values of $a^{0}(x)$.

To give more intuition on how the porous geometry varies, we plot $\Omega_{\varepsilon}$ in Figure 5 . However, we do not follow the definition (3) as it would create artificial corners at the boundaries of neighboring tiles, since the channel widths would not match exactly. Instead, we define the porous geometry by (see also [3] for details) $\Omega_{\varepsilon}=\Omega \backslash \cup_{k \in \mathbb{Z}^{d}}\left\{\varepsilon(k+\varphi(\epsilon k+\epsilon y, y)) \mid y \in Y_{\mathrm{S}}\right\}$.

At the macro scale (see (6)) we set the force field $f \equiv 0$. For the boundary conditions, we set $g_{\mathrm{D}}(x)=0$ for $x \in \Gamma_{\mathrm{D}}=(-3,3) \times\{-2\}$ and $\Gamma_{\mathrm{N}}(x)=1$ for $x \in \Gamma_{\mathrm{N}}=(-3,3) \times\{2\}$. The remaining two edges $\{-3\} \times(-2,2)$ and $\{3\} \times(-2,2)$ are assumed to be connected periodically. We choose such boundary conditions to provide high regularity of $p^{0}$ so that we can test higher order finite elements with uniform macro meshes. A sketch of the exact solution $p^{0}$ and the related velocity field is plotted in Figure 6.

Macroscopic meshes. In all experiments we consider uniform macroscopic meshes. The coarsest macro has 24 elements and we consider 6 additional meshes, where each new mesh is a uniform refinement of the previous one. See Figure 7. 


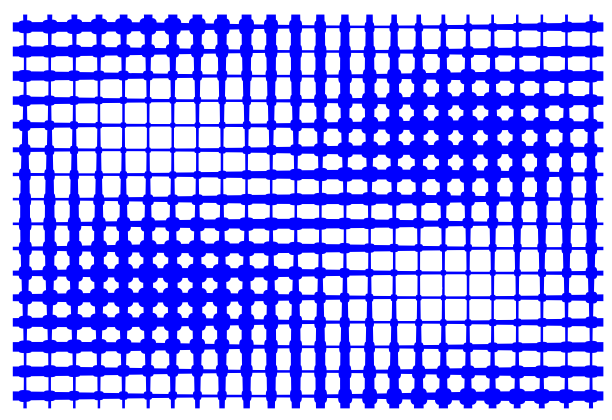

Figure 5: A sketch of $\Omega_{\varepsilon}$ for $\varepsilon=1 / 4$.


Figure 6: Solution $p^{0}$ with contours (left), magnitude of the velocity field $\left|a^{0}(x) \nabla p^{0}\right|$ with streamlines (right).
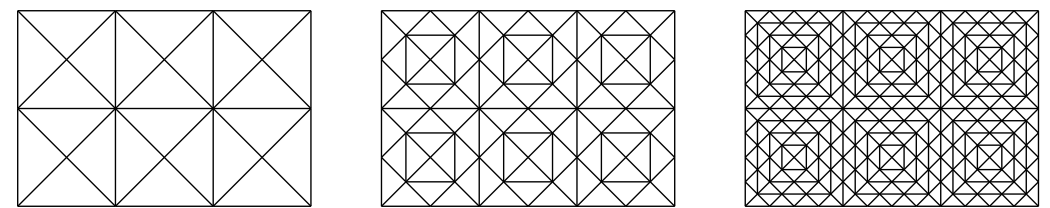

Figure 7: First three (out of 7) uniform macro meshes $\mathcal{T}_{H}$ considered in the experiments.

Uniform micro meshes. For testing purposes we considered different micro meshes. The coarsest micro mesh is depicted in Figure 8(left) and is denoted $\mathcal{T}_{h}^{0}$. It contains 2216 elements and the corresponding degrees of freedom for the micro problems are $N_{\text {mic }}=10150$. We define a sequence of meshes $\mathcal{T}_{h}^{0}, \mathcal{T}_{h}^{1}, \mathcal{T}_{h}^{2}, \ldots$, where each new mesh is a uniform refinement of the previous one.

Adaptive micro meshes. Since $Y_{\mathrm{F}}$ contains re-entrant corners, micro solutions can benefit from meshes that are adapted to the geometry. We applied the adaptive micro mesh refinement from [5]. Starting with the coarsest uniform mesh $\mathcal{T}_{h}^{0}$ we considered the extreme geometries (see Figure 4). We repeatedly map the current micro mesh to the extreme geometries, solve the micro problems, compute the residuals for every element and mark and refine the micro mesh according to the maximum residual over all four geometries. During this adaptive process we stopped the refinement when we reach successively $20000,40000,80000, \ldots$ degrees of freedom and we denoted by $\mathcal{T}_{h}^{\text {ad, },}, \mathcal{T}_{h}^{\text {ad,2 }}, \mathcal{T}_{h}^{\text {ad, }, 3}, \ldots$ the obtained micro meshes.
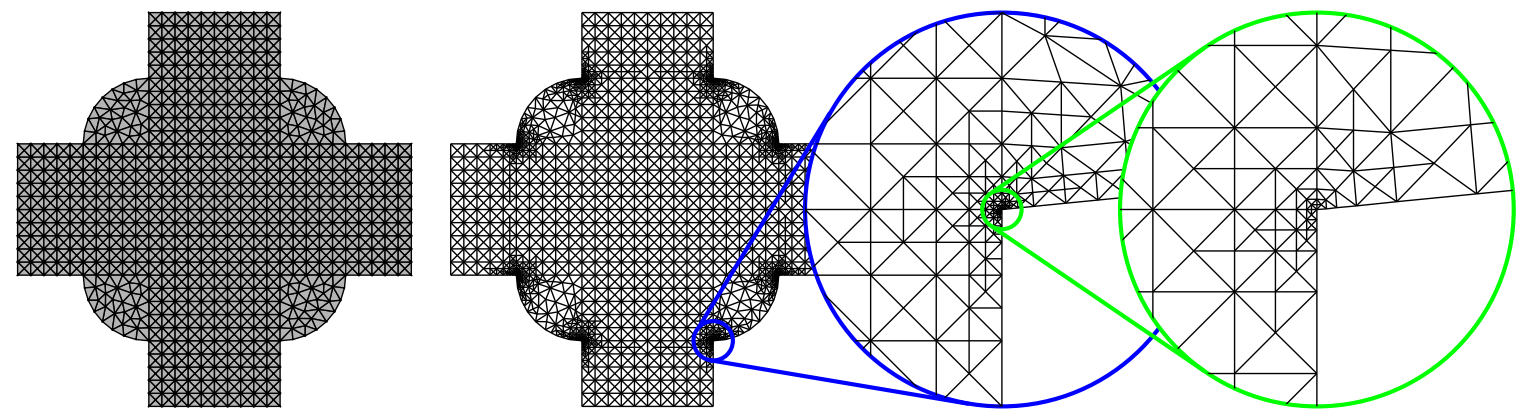

Figure 8: The coarsest uniform micro mesh $\mathcal{T}_{h}^{0}$ (left) and the first adaptive micro mesh $\mathcal{T}_{h}^{\text {ad,1 }}$ with a zoomed in interesting part (right). 
RB offline. For all different micro meshes we used the same settings in the RB offline procedure. For discretization we used the stable Taylor-Hood elements $\mathcal{P}_{2} / \mathcal{P}_{1}$, that is, $l=1$. We ran the reduced basis over the parametric space $x \in \Omega$, which was sampled randomly by $\Xi_{\text {train }}$ of size 10000 . Setting the tolerance to $\varepsilon_{\text {tol }}=10^{-5}$ we obtained the RB space with $N_{1}, N_{2} \approx 55$ basis functions in all the cases.

Micro error. We first tested the influence of the micro mesh on the overall error. To minimize the RB error we took the complete reduced basis $N_{1}, N_{2} \approx 50$. An experiment with $\mathcal{P}_{1}$ macro elements is shown in Figure 9, where the saturation of the micro error is visible for all the uniform meshes $\mathcal{T}_{h}^{0}, \ldots, \mathcal{T}_{h}^{4}$. As expected, with finer micro meshes the error is saturated at a lower value. It is remarkable that with the coarsest adaptive micro mesh we get a smaller micro error than with the finest uniform micro mesh that we considered. We emphasize that the online computation time is independent of the degrees of freedom of the micro mesh.

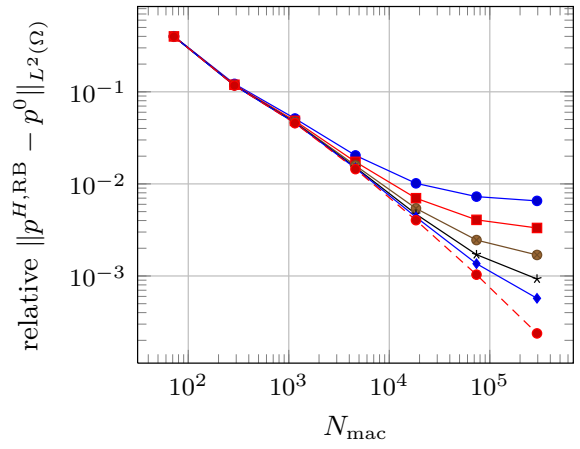

Uniform micro meshes

$\longrightarrow \mathcal{T}_{h}^{0}, N_{\text {mic }}=10150$

$\rightarrow \mathcal{T}_{h}^{1}, N_{\text {mic }}=40246$

$-\mathcal{T}_{h}^{2}, N_{\text {mic }}=160270$

* $\mathcal{T}_{h}^{3}, N_{\text {mic }}=639646$

$\longrightarrow \mathcal{T}_{h}^{4}, N_{\text {mic }}=2555710$

Adaptive micro mesh

$\bullet \mathcal{T}_{h}^{\mathrm{ad}, 1}, N_{\text {mic }}=21462$

Figure 9: Convergence rates of $p^{H, \mathrm{RB}}$ with $\mathcal{P}_{1}$ macro FE and uniform macro refinement with different micro meshes. The RB is set to maximum: $N_{1}, N_{2} \approx 50$.

We repeated the same experiment but this time with $\mathcal{P}_{2}$ and $\mathcal{P}_{3}$ macro elements and only the adaptive micro meshes $\mathcal{T}_{h}^{\text {ad, } 1}, \ldots, \mathcal{T}_{h}^{\text {ad, }, 5}$. The convergence rates are shown in Figure 10.
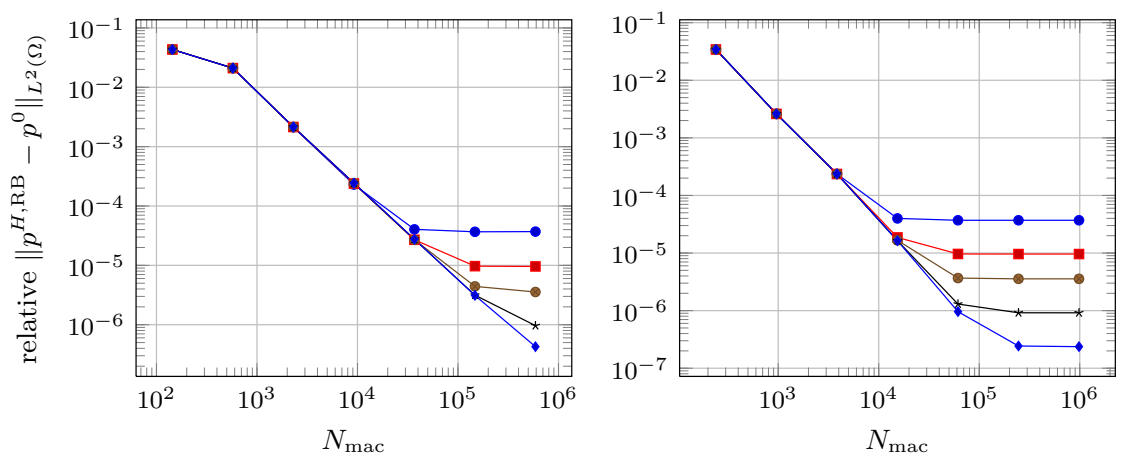

Adaptive micro mesh

$\longrightarrow \mathcal{T}_{h}^{\mathrm{ad}, 1}, N_{\text {mic }}=21462$

$-\mathcal{T}_{h}^{\mathrm{ad}, 2}, N_{\text {mic }}=44156$

$\rightarrow \mathcal{T}_{h}^{\text {ad, } 3}, N_{\text {mic }}=84249$

$\longrightarrow \mathcal{T}_{h}^{\mathrm{ad}, 4}, N_{\text {mic }}=177689$

$\rightarrow \mathcal{T}_{h}^{\mathrm{ad}, 5}, N_{\text {mic }}=327032$

Figure 10: Convergence rates of $p^{H, \mathrm{RB}}$ with $\mathcal{P}_{2}$ (left) and $\mathcal{P}_{3}$ (right) macro FE and uniform macro refinement with different micro meshes. The RB is set to maximum: $N_{1}, N_{2} \approx 50$.

RB error. We have seen that we can expect the best results with the finest adapted micro mesh $\mathcal{T}_{h}^{\text {ad, } 5}$. Hence, we choose this micro mesh and run the multiscale method with uniform refinement on the macro scale and varying number of RB functions $N_{\mathrm{RB}}=N_{1}=N_{2}$. We monitor the relative macroscopic error in the pressure. For $\mathcal{P}_{1}$ macro elements, the resulting convergence rates are plotted in Figure 11. We see that already taking $N_{\mathrm{RB}}=7$ is sufficient for the finest macro mesh.

We next choose $\mathcal{P}_{3}$ macro elements and repeat the experiment. From Figure 12 we see that for $N_{\mathrm{RB}}=25$ the error is saturated even for the finest macro mesh and the micro error becomes dominant.

Conservation of mass. One of the desirable properties of a DG method is conservation of mass, especially in time-dependent problems. For every interior edge $e \in \mathcal{E}_{\text {int }}$ the numerical flux is defined as $\hat{\sigma}_{K}=\left\{\Pi_{a_{\mathrm{RB}}}\left(\nabla p^{H, \mathrm{RB}}\right)\right\}-\sigma \llbracket p^{H, \mathrm{RB}} \rrbracket$. These fluxes are conservative, that is, for element $K \in \mathcal{T}_{H}$ with no boundary edges we have the conservation property $\int_{\partial K} \hat{\sigma}_{K} \mathrm{~d} s=$ 


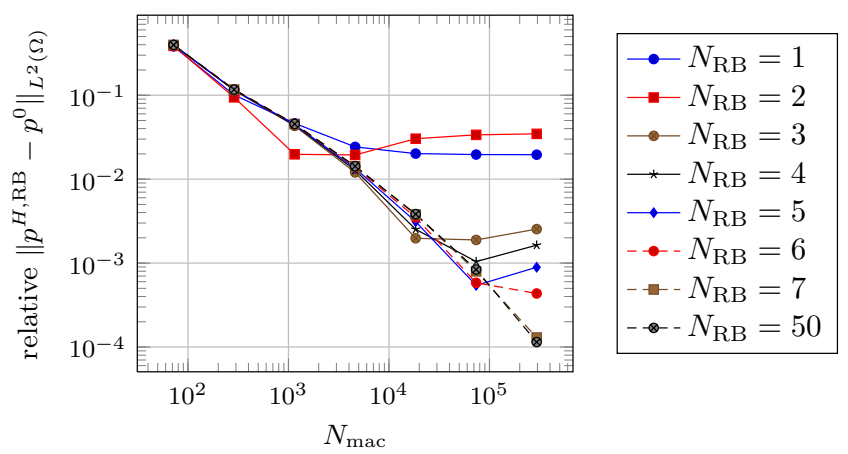

Figure 11: Convergence rates of $p^{H, \mathrm{RB}}$ with $\mathcal{P}_{1}$ macro FE, micro mesh $\mathcal{T}_{h}^{\text {ad, }, 5}$, and a varying number of RB functions $N_{\mathrm{RB}}=N_{1}=N_{2}$.



Figure 12: Left: Convergence rates of $p^{H, \mathrm{RB}}$ with $\mathcal{P}_{1}$ macro FE, micro mesh $\mathcal{T}_{h}^{\text {ad,5 }}$, and a varying number of RB functions $N_{\mathrm{RB}}=N_{1}=N_{2}$. Right: Convergence of the maximal residual in the offline greedy algorithm for the reduced basis.

$\int_{K} f \mathrm{~d} x$. The flux over boundary edges is treated differently. To express the conservation of mass for any element, we take any $K \in \mathcal{T}_{H}$ and $e \in \partial K$ and define

$$
F_{e}^{K}= \begin{cases}\int_{e}\left(\left\{\Pi_{a^{\mathrm{RB}}}\left(\nabla p^{H, \mathrm{RB}}\right)\right\}-\sigma_{e} \llbracket p^{H, \mathrm{RB}} \rrbracket\right) \mathbf{n} \mathrm{d} s & \text { if } e \in \mathcal{E}_{\mathrm{int}}, \\ \int_{e}\left(\left\{\Pi_{a^{\mathrm{RB}}}\left(\nabla p^{H, \mathrm{RB}}\right)\right\}-\sigma_{e} \llbracket p^{H, \mathrm{RB}}-g_{\mathrm{D}} \rrbracket\right) \mathbf{n} \mathrm{d} s & \text { if } e \in \mathcal{E}_{\mathrm{D}}, \\ \int_{e} g_{\mathrm{N}} \mathrm{d} s & \text { if } e \in \mathcal{E}_{\mathrm{N}} .\end{cases}
$$

It is then guaranteed that

$$
\sum_{e \in \partial K} F_{e}^{K}=\int_{K} f \mathrm{~d} x \quad \forall K \in \mathcal{T}_{H} .
$$

We examined the conservation property (56) numerically by computing the left hand side value of (56) for every element in Figure 13(top). Since $f \equiv 0$ in our experiment, we expect these values to be very close to zero, which seems to hold (up to round-off errors). Evaluation of the same quantity for a multiscale method with continuous macro elements (the RB-DS-FE-HMM from [5]) results in values whose absolute value are significantly larger, see Figure 13(bottom). Compared to continuous FE, where reconstruction techniques are used to postprocess the solution to be conservative, with a SIP-DG method such properties are valid without any additional procedure.

5.2. A 3D problem. We now consider a three-dimensional example. The macroscopic domain will be a filtration bottle given by $\Omega=\left\{x \in \mathbb{R}^{3}: x_{1} \in(-1,1), x_{2}^{2}+x_{3}^{2} \leq\right.$ $\left.g\left(x_{1}\right)^{2}\right\}$, where $g(r)=0.2$ for $r<-1 / 2, g(r)=0.6$ for $r>1 / 2$, and $g(r)=0.2 \sin (\pi r)+0.4$ otherwise (see Figure 14).

The microscopic domains $Y_{\mathrm{F}}^{x}$ are defined as unions of three ellipsoidal cylinders. See Figure 15 for a sketch of the following definition. We define

$$
Y_{\mathrm{F}}^{x}=\left\{y \in Y: \min \left\{\frac{y_{1}^{2}}{\mu_{1}^{2}}+\frac{y_{2}^{2}}{\mu_{2}^{2}}, \frac{y_{1}^{2}}{\mu_{1}^{2}}+\frac{y_{3}^{2}}{\mu_{3}^{2}}, \frac{y_{2}^{2}}{\mu_{2}^{2}}+\frac{y_{3}^{2}}{\mu_{3}^{2}}\right\}<0.8^{2}\right\},
$$



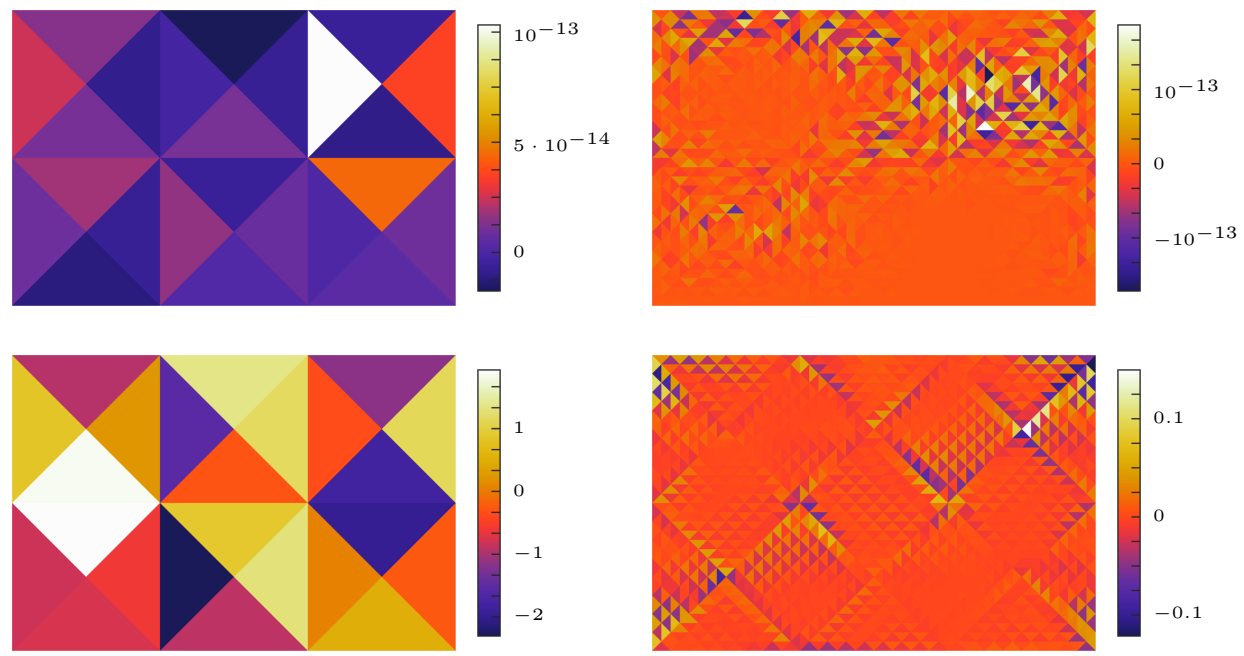

Figure 13: Comparison of conservations of the proposed numerical method (top) and the RB-DS-FE-HMM (see [5]) that uses continuous FE on the macro scale (bottom). Plotted are the values of the left hand sides of (56) that were computed for two different macro meshes (left vs. right), $\mathcal{P}_{1}$ macro elements, micro mesh $\mathcal{T}_{h}^{\text {ad, }, 5}$, and $N_{1}=N_{2}=7$.
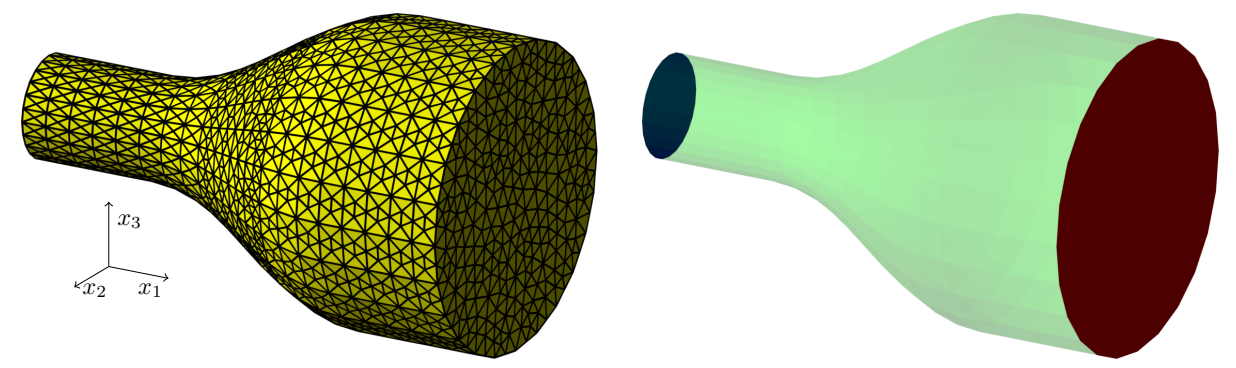

Figure 14: Macroscopic domain $\Omega$ and mesh $\mathcal{T}_{H}$ (left). Boundary conditions (right): Neumann inflow (blue), zero Dirichlet (red), zero Neumann (transparent green).

where the functions $\mu_{1}, \mu_{2}, \mu_{3}$ depend on $x$ (see (58)). The reference micro domain corresponds to $\mu_{1}=\mu_{2}=\mu_{3}=1 / 4$. Figure 15 illustrates how we can cut $Y_{\mathrm{F}}$ with 6 planes such that $Y_{\mathrm{F}}^{x}$ can be obtained stretching or contracting the planes in each direction. That is, we can divide $Y_{\mathrm{F}}$ into 7 regions such that an implicitly defined $\varphi(x, \cdot)$ will be affine in each region. To avoid degenerate cases we will allow only $0<\mu_{1}, \mu_{2}, \mu_{3}<1 / 2$. We set

$$
\begin{aligned}
& \mu_{1}(x)=1 / 4+\sin \left(x_{1}+2 x_{2}+3 x_{3}\right) / 8, \\
& \mu_{2}(x)=1 / 4+\sin \left(-2 x_{1}+x_{2}-3 x_{3}\right) / 8, \\
& \mu_{3}(x)=1 / 4+\sin \left(3 x_{1}-x_{2}+x_{3}\right) / 8 .
\end{aligned}
$$

In the reduced basis offline algorithm we used tolerance $\varepsilon_{\text {tol }}=0.0005$ and the training set was random selection of points from $\Omega$ with $\left|\Xi_{\text {train }}\right|=65^{3}$. The resulting sizes of RB were $N_{1}=59, N_{2}=61$, and $N_{3}=58$. In Figure 16 we sketched a plot of some pressure isosurfaces for the pressure solution computed with the multiscale numerical method.

\section{Conclusion}

We have presented a multiscale FE method for Stokes flow in porous media. The method uses a discontinuous Galerkin discretization of the effective Darcy problem at the macroscopic scale. The effective permeability is recovered at every quadrature point of the macroscopic using local porous geometry. We applied the reduced basis method for a fast and accurate approximation of the permeability, allowing for a fast (mesh independent) computation of the permeability in an online stage. We discussed a priori error analysis and provided a priori convergence rates for the proposed multiscale method. Various sources of discretisation error have also been studied numerically and the performance and accuracy of the method has also been illustrated. The method allows for further generalizations. In particular, tools 


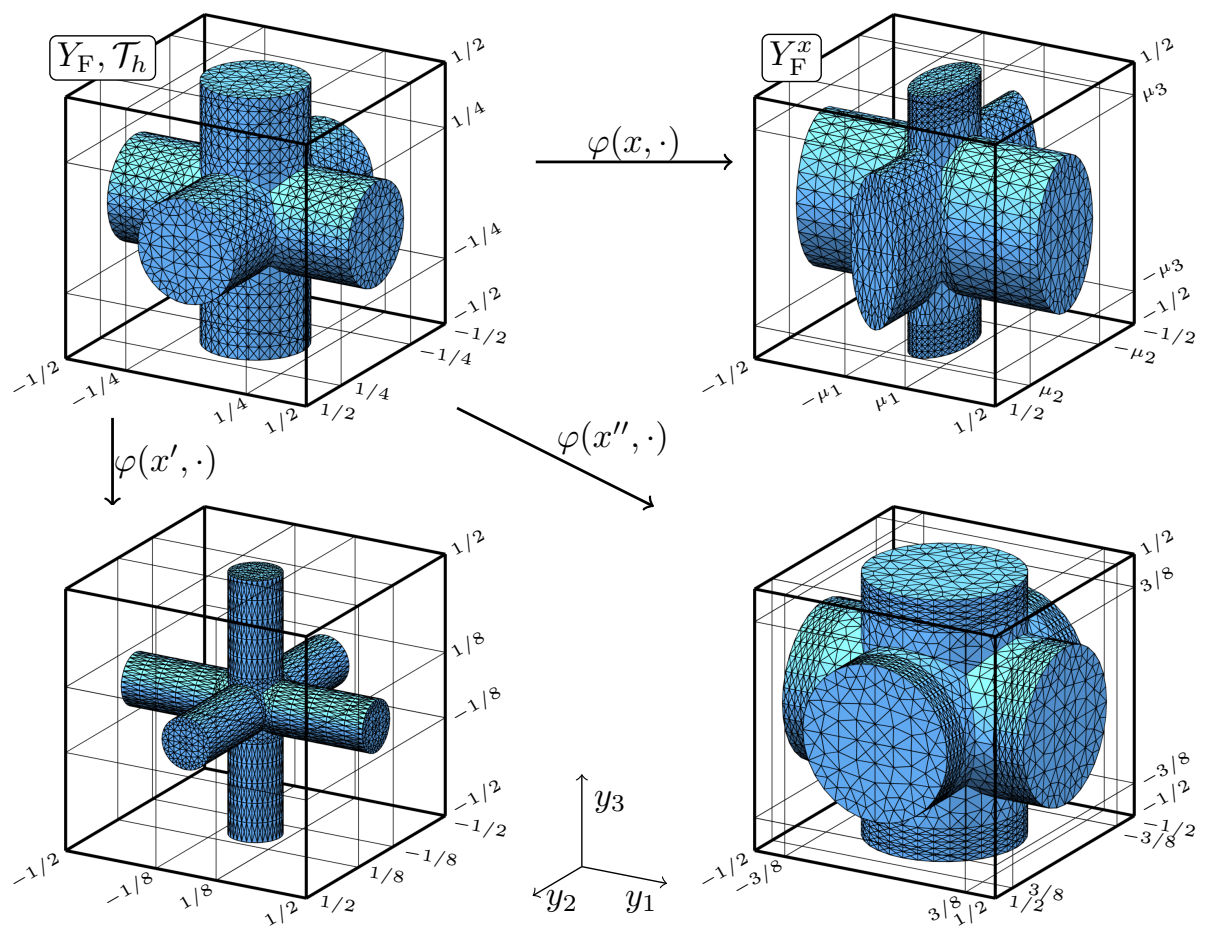

Figure 15: Reference micro domain $Y_{\mathrm{F}}$ and mesh $\mathcal{T}_{h}$ (upper left corner) and some local geometries $Y_{\mathrm{F}}^{x}$.

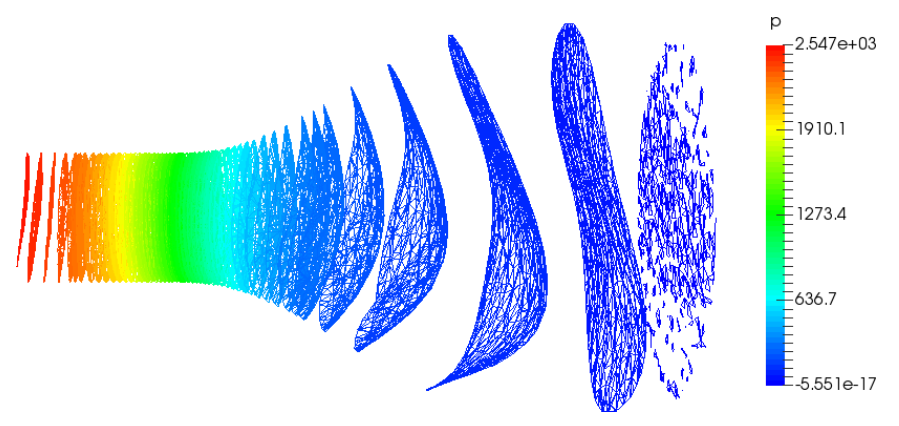

Figure 16: A plot of some pressure isosurfaces of $p^{H, \mathrm{RB}}$. The isosurface of zero value (rightmost) is scattered since the Dirichlet boundary conditions are enforced weakly.

developed for single scale DG-FEM such as adaptive mesh refinement or hp-adaptivity, can be applied on the macro scale without changing the micro solver.

\section{References}

[1] AbDulle, A. A priori and a posteriori error analysis for numerical homogenization: a unified framework. Ser. Contemp. Appl. Math. CAM 16 (2011), 280-305.

[2] AbDulle, A. Discontinuous Galerkin finite element heterogeneous multiscale method for elliptic problems with multiple scales. Math. Comp. 81, 278 (2012), 687-713.

[3] Abdulle, A., And Budáč, O. An adaptive finite element heterogeneous multiscale method for Stokes flow in porous media. Multiscale Model. Simul. 13 (2015), 256-290.

[4] AbDulle, A., AND BudÁČ, O. A Petrov-Galerkin reduced basis approximation of the Stokes equation in parameterized geometries. C. R. Math. Acad. Sci. Paris 353, 7 (2015), 641-645.

[5] Abdulle, A., And Budáč, O. A reduced basis finite element heterogeneous multiscale method for Stokes flow in porous media. submitted for publication, 2015.

[6] Abdulle, A., E, W., Engquist, B., and Vanden-Eijnden, E. The heterogeneous multiscale method. Acta Numer. 21 (2012), 1-87.

[7] Abdulle, A., And Huber, M. E. Discontinuous Galerkin finite element heterogeneous 
multiscale method for advection-diffusion problems with multiple scales. Numer. Math. 126, 4 (2014), 589-633.

[8] Abdulle, A., And Nonnenmacher, A. A short and versatile finite element multiscale code for homogenization problems. Comput. Methods Appl. Mech. Engrg. 198, 37-40 (2009), 2839-2859.

[9] Allaire, G. Homogenization of the Stokes flow in a connected porous medium. Asymptot. Anal. 2, 3 (1989), 203-222.

[10] Alyaev, S., Keilegavlen, E., and Nordbotten, J. M. Analysis of control volume heterogeneous multiscale methods for single phase flow in porous media. Multiscale Model. Simul. 12, 1 (2014), 335-363.

[11] Arnold, D. N., Brezzi, F., Cockburn, B., and Marini, L. D. Unified analysis of discontinuous Galerkin methods for elliptic problems. SIAM J. Numer. Anal. 39, 5 (2002), 1749-1779.

[12] Barrault, M., Maday, Y., Nguyen, N.-C., and Patera, A. T. An 'empirical interpolation method': Application to efficient reduced-basis discretization of partial differential equations. C. R. Math. Acad. Sci. Paris Ser.I 339 (2004), 667-672.

[13] Brown, D. L., Efendiev, Y., And Hoang, V. H. An efficient hierarchical multiscale finite element method for Stokes equations in slowly varying media. Multiscale Model. Simul. 11, 1 (2013), 30-58.

[14] Brown, D. L., Popov, P., And Efendiev, Y. On homogenization of Stokes flow in slowly varying media with applications to fluid-structure interaction. GEM Int. J. Geomath. 2, 2 (2011), 281-305.

[15] Chen, L., And ZhAng, C.-S. AFEM@Matlab: a matlab package of adaptive finite element methods. Tech. rep., Department of Mathematics, University of Maryland at College Park, 2006.

[16] Houston, P., Schwab, C., And Süli, E. Discontinuous $h p$-finite element methods for advection-diffusion-reaction problems. SIAM J. Numer. Anal. 39, 6 (2002), 2133-2163.

[17] Huynh, D. B. P., Knezevic, D. J., Chen, Y., Hesthaven, J. S., and Patera, A. T. A natural-norm successive constraint method for inf-sup lower bounds. Comput. Methods Appl. Mech. Engrg. 199, 29 (2010), 1963-1975.

[18] Notay, Y. An aggregation-based algebraic multigrid method. Electron. Trans. Numer. Anal. 37, 6 (2010), 123-146.

[19] Peters, J., Reichelt, V., And Reusken, A. Fast iterative solvers for discrete Stokes equations. SIAM J. Sci. Comput. 27, 2 (2005), 646-666.

[20] Pierce, N. A., And Giles, M. B. Adjoint recovery of superconvergent functionals from PDE approximations. SIAM Rev. 42, 2 (2000), 247-264.

[21] Quillen, P. D. Generalizations of an inverse free Krylov subspace method for the symmetric generalized eigenvalue problem. PhD thesis, University of Kentucky, 2005.

[22] SÁnchez-PAlencia, E. Non-homogeneous media and vibration theory, vol. 127 of Lecture Notes in Phys. Springer, 1980.

[23] Sandström, C., Larsson, F., Runesson, K., And Johansson, H. A two-scale finite element formulation of Stokes flow in porous media. Comput. Methods Appl. Mech. Engrg. 261-262 (2013), 96-104.

[24] TARTAR, L. Incompressible fluid flow in a porous medium-convergence of the homogenization process. Vol. 127 of Lecture Notes in Phys. [22], 1979, ch. Appendix, pp. 368-377.

[25] Warburton, T., and Hesthaven, J. S. On the constants in hp-finite element trace inverse inequalities. Comp. Meth. Appl. Mech. Eng. 192, 25 (2003), 2765-2773. 